\title{
Chapter 7 \\ Linear Canonical Domains and Degrees of Freedom of Signals and Systems
}

\author{
Figen S. Oktem and Haldun M. Ozaktas
}

\begin{abstract}
We discuss the relationships between linear canonical transform (LCT) domains, fractional Fourier transform (FRT) domains, and the space-frequency plane. In particular, we show that LCT domains correspond to scaled fractional Fourier domains and thus to scaled oblique axes in the space-frequency plane. This allows LCT domains to be labeled and monotonically ordered by the corresponding fractional order parameter and provides a more transparent view of the evolution of light through an optical system modeled by LCTs. We then study the number of degrees of freedom of optical systems and signals based on these concepts. We first discuss the bicanonical width product (BWP), which is the number of degrees of freedom of LCT-limited signals. The BWP generalizes the space-bandwidth product and often provides a tighter measure of the actual number of degrees of freedom of signals. We illustrate the usefulness of the notion of BWP in two applications: efficient signal representation and efficient system simulation. In the first application we provide a sub-Nyquist sampling approach to represent and reconstruct signals with arbitrary space-frequency support. In the second application we provide a fast discrete LCT (DLCT) computation method which can accurately compute a (continuous) LCT with the minimum number of samples given by the BWP. Finally, we focus on the degrees of freedom of first-order optical systems with multiple apertures. We show how to explicitly quantify the degrees of freedom of such systems, state conditions for lossless transfer through the system and analyze the effects of lossy transfer.
\end{abstract}

F.S. Oktem $(\bowtie)$

Department of Electrical and Electronics Engineering, Middle East Technical University, 06800 Cankaya, Ankara, Turkey

e-mail: figeno@metu.edu.tr

H.M. Ozaktas

Department of Electrical Engineering, Bilkent University, 06800 Bilkent, Ankara, Turkey

e-mail: haldun@ee.bilkent.edu.tr 


\subsection{Introduction}

Optical systems involving thin lenses, sections of free space in the Fresnel approximation, sections of quadratic graded-index media, and arbitrary combinations of any number of these are referred to as first-order optical systems or quadratic-phase systems [1-5]. Mathematically, such systems can be modeled as linear canonical transforms (LCTs), which form a three-parameter family of integral transforms [5, 6]. The LCT family includes the Fourier and fractional Fourier transforms (FRTs), coordinate scaling, chirp multiplication, and convolution operations as its special cases.

One of the most important concepts in Fourier analysis is the concept of the frequency (or Fourier) domain. This domain is understood to be a space where the frequency representation of the signal lives. Likewise, fractional Fourier domains are well understood to correspond to oblique axes in the space-frequency plane (phase space) [5, 7]. By analogy with this concept, the term linear canonical domain has been used in several papers to refer to the domain of the LCT representation of a signal [8-16]. Because LCTs are characterized by three independent parameters, LCT domains populate a three-parameter space, which makes them hard to visualize. In this chapter, we discuss the relationships between LCT domains, FRT domains, and the space-frequency plane. In particular, we show that each LCT domain corresponds to a scaled FRT domain, and thus to a scaled oblique axis in the space-frequency plane. Based on this many-to-one association of LCTs with FRTs, LCT domains can be labeled and monotonically ordered by the corresponding fractional order parameter, instead of their usual three parameters which do not directly lend to a natural ordering. This provides a more transparent view of the evolution of light through an optical system modeled by LCTs.

Another important concept is the number of degrees of freedom. For simplicity we focus on one-dimensional signals and systems, though most of our results can be generalized to higher dimensions in a straightforward manner. We first discuss the bicanonical width product, which is the number of degrees of freedom of LCT-limited signals. The conventional space-bandwidth product is of fundamental importance in signal processing and information optics because of its interpretation as the number of degrees of freedom of space- and band-limited signals [5, 17-32]. If, instead, a set of signals is highly confined to finite intervals in two arbitrary LCT domains, the space-frequency (phase space) support is a parallelogram. The number of degrees of freedom of this set of signals is given by the area of this parallelogram, which is equal to the BWP, which is usually smaller than the conventional spacebandwidth product. The BWP, which is a generalization of the space-bandwidth product, often provides a tighter measure of the actual number of degrees of freedom, and allows us to represent and process signals with fewer samples.

We illustrate the usefulness of the bicanonical width product in two applications: efficient signal representation and efficient system simulation. First, we show how to represent and reconstruct signals with arbitrary time- or space-frequency support, using fewer samples than required by the classical Shannon-Nyquist sampling 
theorem. Although the classical approach is optimal for band-limited signals, it is in general suboptimal for representing signals with a known space-frequency support. Based on the LCT sampling theorem, we provide a sub-Nyquist approach to represent signals with arbitrary space-frequency support. This approach geometrically amounts to enclosing the support with the smallest possible parallelogram, as opposed to enclosing it with a rectangle as in the classical approach. The number of samples required for reconstruction is given by the BWP, which is smaller than the number of samples required by the classical approach.

As a second application, we provide a fast discrete LCT (DLCT) computation method which can accurately compute a (continuous) LCT with the minimum number of samples given by the bicanonical width product. Hence the bicanonical width product is also a key parameter in fast discrete computation of LCTs, and hence in efficient and accurate simulation of optical systems.

Lastly, we focus on the degrees of freedom of apertured optical systems, which here refers to systems consisting of an arbitrary sequence of thin lenses and apertures separated by sections of free space. We define the space-frequency window (phase-space window) and show how it can be explicitly determined for such a system. Once the space-frequency window of the system is determined, the area of the window gives the maximum number of degrees of freedom that can be supported by the system. More significantly, it specifies which signals can pass through the system without information loss; the signal will pass losslessly if and only if the space-frequency support of the signal lies completely within this window. When it does not, the parts that lie within the window pass and the parts that lie outside of the window are blocked, a result which is valid to a good degree of approximation for most systems of practical interest. These intuitive results provide insight and guidance into the behavior and design of systems involving multiple apertures and can help minimize information loss.

In the next section, some preliminary material will be reviewed. In Sect. 7.3 we establish the relationships between LCT domains, FRT domains, and the spacefrequency plane $[33,34]$. The relationships between the space-frequency support, the bicanonical width product, and the number of degrees of freedom of signals is the subject of Sect.7.4 [33-35]. We then provide a sub-Nyquist approach to represent signals with arbitrary space-frequency support in Sect. 7.5, which requires the number of samples to be equal to the bicanonical width product $[33,34,36]$. In Sect. 7.6 we review a fast DLCT computation method that works with this minimum number of samples [35]. Section 7.7 discusses how to explicitly quantify the degrees of freedom of optical systems with apertures and analyzes lossless and lossy transfer through them $[33,37]$. We conclude in Sect. 7.8.

While in this chapter we usually refer to the independent variable in our signals as "space" and speak of the "space-frequency" plane due to the development of many of these concepts in an optical context, virtually all of our results are also valid when the independent variable is "time" or when we speak of the "time-frequency" plane. 


\subsection{Background}

In this section we review some preliminary material that will be used throughout the chapter. This includes the definition and properties of LCTs and FRTs, the Iwasawa decomposition, space-frequency distributions, and the LCT sampling theorem.

\subsubsection{Linear Canonical Transforms}

Optical systems involving thin lenses, sections of free space in the Fresnel approximation, sections of quadratic graded-index media, and arbitrary combinations of any number of these are referred to as first-order optical systems or quadratic-phase systems. Mathematically, such systems can be modeled as LCTs. The output light field $f_{\mathbf{T}}(u)$ of a quadratic-phase system is related to its input field $f(u)$ through $[5,6]$

$$
\begin{aligned}
f_{\mathbf{T}}(u) & \equiv\left(\mathcal{C}_{\mathbf{T}} f\right)(u) \equiv \int_{-\infty}^{\infty} C_{\mathbf{T}}\left(u, u^{\prime}\right) f\left(u^{\prime}\right) d u^{\prime}, \\
C_{\mathbf{T}}\left(u, u^{\prime}\right) & \equiv \sqrt{\frac{1}{B}} e^{-i \pi / 4} e^{i \pi\left(\frac{D}{B} u^{2}-2 \frac{1}{B} u u^{\prime}+\frac{A}{B} u^{\prime 2}\right)},
\end{aligned}
$$

for $B \neq 0$, where $\mathcal{C}_{\mathbf{T}}$ is the unitary LCT operator with parameter matrix $\mathbf{T}=$ $[A B ; C D]$ with $A D-B C=1$. In the trivial case $B=0$, the LCT is defined simply as $f_{\mathbf{T}}(u) \equiv \sqrt{D} \exp \left[i \pi C D u^{2}\right] f(D u)$. Sometimes the three real parameters $\alpha=D / B$, $\beta=1 / B, \gamma=A / B$ are used instead of the unit-determinant matrix $\mathbf{T}$ whose elements are $A, B, C, D$. (One of the four matrix parameters is redundant because of the unit-determinant condition.) These two sets of parameters are equivalent and either set of parameters can be obtained from the other $[5,6]$ :

$$
\mathbf{T}=\left[\begin{array}{ll}
A & B \\
C & D
\end{array}\right]=\left[\begin{array}{cc}
\gamma / \beta & 1 / \beta \\
-\beta+\alpha \gamma / \beta & \alpha / \beta
\end{array}\right]
$$

The transform matrix $\mathbf{T}$ is useful in the analysis of optical systems because if several systems are cascaded, the overall system matrix can be found by multiplying the corresponding matrices.

The Fourier transform, FRT (propagation through quadratic graded-index media), coordinate scaling (imaging), chirp multiplication (passage through a thin lens), and chirp convolution (Fresnel propagation in free space) are some of the special cases of LCTs.

The $a$ th-order FRT [5] of a function $f(u)$, denoted by $f_{a}(u)$, can be defined as

$$
f_{a}(u) \equiv\left(\mathcal{F}^{a} f\right)(u) \equiv \int_{-\infty}^{\infty} K_{a}\left(u, u^{\prime}\right) f\left(u^{\prime}\right) d u^{\prime},
$$




$$
\begin{gathered}
K_{a}\left(u, u^{\prime}\right) \equiv A_{\phi} e^{i \pi\left(\cot \phi u^{2}-2 \csc \phi u u^{\prime}+\cot \phi u^{\prime 2}\right)}, \\
A_{\phi}=\sqrt{1-i \cot \phi}, \quad \phi=a \pi / 2
\end{gathered}
$$

when $a \neq 2 k$, and $K_{a}\left(u, u^{\prime}\right)=\delta\left(u-u^{\prime}\right)$ when $a=4 k$, and $K_{a}\left(u, u^{\prime}\right)=\delta\left(u+u^{\prime}\right)$ when $a=4 k \pm 2$, where $k$ is an integer. The FRT operator $\mathcal{F}^{a}$ is additive in index: $\mathcal{F}^{a_{2}} \mathcal{F}^{a_{1}}=\mathcal{F}^{a_{2}+a_{1}}$ and reduces to the Fourier transform (FT) and identity operators for $a=1$ and $a=0$, respectively. The FRT is a special case of the LCT with parameter matrix

$$
\mathbf{F}^{a}=\left[\begin{array}{rr}
\cos (a \pi / 2) & \sin (a \pi / 2) \\
-\sin (a \pi / 2) & \cos (a \pi / 2)
\end{array}\right]
$$

differing only by an inconsequential factor: $\mathcal{C}_{\mathbf{F}^{a}} f(u)=e^{-i a \pi / 4} \mathcal{F}^{a} f(u)[5,6]$.

Other than the FRT, another special case of the LCT is multiplication with a chirp function of the form $\exp \left[-i \pi q u^{2}\right]$, which corresponds to a thin lens in optics. The corresponding LCT matrix is given by

$$
\mathbf{Q}_{q}=\left[\begin{array}{cc}
1 & 0 \\
-q & 1
\end{array}\right]
$$

Yet another special case is convolution with a chirp function of the form $e^{-i \pi / 4} \sqrt{1 / r} \exp \left[i \pi u^{2} / r\right]$, which is equivalent to propagation through a section of free space in the Fresnel approximation. The corresponding LCT matrix is given by

$$
\mathbf{R}_{r}=\left[\begin{array}{ll}
1 & r \\
0 & 1
\end{array}\right]
$$

The last special case we consider is the scaling operation, which maps a function $f(u)$ into $\sqrt{1 / M} f(u / M)$ with $M>0$. This is often used to model optical imaging. The transformation matrix is

$$
\mathbf{M}_{M}=\left[\begin{array}{cc}
M & 0 \\
0 & 1 / M
\end{array}\right]
$$

\subsubsection{Iwasawa Decomposition}

An arbitrary LCT can be decomposed into an FRT followed by scaling followed by chirp multiplication [5, 34]:

$$
\mathbf{T}=\left[\begin{array}{ll}
A & B \\
C & D
\end{array}\right]=\left[\begin{array}{cc}
1 & 0 \\
-q & 1
\end{array}\right]\left[\begin{array}{cc}
M & 0 \\
0 & \frac{1}{M}
\end{array}\right]\left[\begin{array}{cc}
\cos \phi & \sin \phi \\
-\sin \phi & \cos \phi
\end{array}\right]
$$


The three matrices, respectively, correspond to the transformation matrices of chirp multiplication with parameter $q$ (multiplication by $\exp \left(-i \pi q u^{2}\right)$ ), coordinate scaling with factor $M>0$ (mapping of $f(u)$ into $\sqrt{1 / M} f(u / M)$ ), and $a$ th order FRT with $\phi=a \pi / 2$ (transformation of $f(u)$ into $f_{a}(u)$ ). The decomposition can be written more explicitly in terms of the LCT and FRT domain representations of the signal as

$$
f_{\mathbf{T}}(u)=\exp \left(-i \pi q u^{2}\right) \sqrt{\frac{1}{M}} f_{a}\left(\frac{u}{M}\right) .
$$

This decomposition is a special case of the Iwasawa decomposition [38-40]. (For a discussion of the implications of this decomposition to the propagation of light through first-order optical systems, see [34, 41]. For a discussion of the implications for sampling optical fields, see $[42,43]$.) By appropriately choosing the three parameters $a, M, q$, the above equality can be satisfied for any $\mathbf{T}=[A B ; C D]$ matrix. Solving for $a, M, q$ in (7.8), we obtain the decomposition parameters in terms of the matrix entries $A, B, C, D$ :

$$
\begin{aligned}
a & = \begin{cases}\frac{2}{\pi} \arctan \left(\frac{B}{A}\right), & \text { if } A \geq 0 \\
\frac{2}{\pi} \arctan \left(\frac{B}{A}\right)+2, & \text { if } A<0\end{cases} \\
M & =\sqrt{A^{2}+B^{2}}, \\
q & = \begin{cases}-\frac{C}{A}-\frac{B / A}{A^{2}+B^{2}}, & \text { if } A \neq 0 \\
-\frac{D}{B}, & \text { if } A=0 .\end{cases}
\end{aligned}
$$

The range of the arctangent lies in $(-\pi / 2, \pi / 2]$.

\subsubsection{Space-Frequency Distributions}

The Wigner distribution (WD) $W_{f}(u, \mu)$ of a signal $f(u)$ is a space-frequency (phase-space) distribution that gives the distribution of signal energy over space and frequency, and is defined as [5, 44-46]:

$$
W_{f}(u, \mu)=\int_{-\infty}^{\infty} f\left(u+u^{\prime} / 2\right) f^{*}\left(u-u^{\prime} / 2\right) e^{-i 2 \pi \mu u^{\prime}} d u^{\prime} .
$$

We refer to the space-frequency region for which the Wigner distribution is considered non-negligible as the space-frequency support of the signal, with the area of this region giving the number of degrees of freedom [5]. A large percentage of the signal energy is confined to the space-frequency support. 
All quadratic-phase systems result in an area-preserving geometric transformation in the $u$ - $\mu$ plane. Explicitly, the WD of $f_{\mathbf{T}}(u)$ can be related to the WD of $f(u)$ by a linear distortion [5]:

$$
W_{f_{\mathbf{T}}}(u, \mu)=W_{f}(D u-B \mu,-C u+A \mu) .
$$

The Jacobian of this coordinate transformation is equal to the determinant of the matrix $\mathbf{T}$, which is unity. Therefore, this coordinate transformation will geometrically distort the support region of the WD but the support area (hence the number of degrees of freedom) will remain unchanged.

\subsubsection{LCT Sampling Theorem}

Just as the LCT is a generalization of the Fourier transform, the LCT sampling theorem $[9,47,48]$ is an extension of the classical sampling theorem. According to the LCT sampling theorem, if a function $f(u)$ has an LCT with parameter $\mathbf{T}$ which has a compact support such that $f_{\mathbf{T}}(u)$ is zero outside the interval $\left[-\Delta u_{\mathbf{T}} / 2, \Delta u_{\mathbf{T}} / 2\right]$, then the function $f(u)$ can be reconstructed from its samples taken at intervals $\delta u \leq 1 /\left(|\beta| \Delta u_{\mathbf{T}}\right)$. The reconstruction formula, which we will refer to as the LCT interpolation formula, is given by

$$
f(u)=\delta u|\beta| \Delta u_{\mathbf{T}} e^{-i \pi \gamma u^{2}} \sum_{n=-\infty}^{\infty} f(n \delta u) e^{i \pi \gamma(n \delta u)^{2}} \operatorname{sinc}\left(\beta \Delta u_{\mathbf{T}}(u-n \delta u)\right) .
$$

This reduces to the classical sampling theorem, and to the FRT sampling theorem [49-54] when the parameter matrix $\mathbf{T}$ is replaced with the associated matrices of the FT and FRT operations.

The background material presented in this section employs dimensionless variables and parameters, for simplicity and purity. We assume that a dimensional normalization has been performed and that the coordinates appearing in the definitions of the FRT, LCT, Wigner distribution, etc., are all dimensionless quantities [5]. In Sect.7.7, however, we will prefer to employ variables with real physical dimensions. There, we will present dimensional counterparts of the background material that we will need. The reader will be able to employ these to obtain dimensional counterparts of other results in this chapter, should the need arise.

\subsection{LCT Domains}

Because LCTs are characterized by three independent parameters, LCT domains populate a three-parameter space, which makes them hard to visualize. In this section, we discuss the relationships between LCT domains, FRT domains, and the 
space-frequency plane. In particular, we show that each LCT domain corresponds to a scaled FRT domain, and thus to a scaled oblique axis in the space-frequency plane. This provides a more transparent view of the evolution of light through an optical system modeled by LCTs.

\subsubsection{Relationship of LCT Domains to the Space-Frequency Plane}

One of the most important concepts in Fourier analysis is the concept of the frequency (or Fourier) domain. This domain is understood to be a space where the frequency representation of the signal lives. Likewise, fractional Fourier domains are well understood to correspond to oblique axes in the space-frequency plane (phase space) [5, 7], since the FRT has the effect of rotating the space-frequency (phase space) representation of a signal. More explicitly, the effect of $a$ th-order fractional Fourier transformation on the Wigner distribution of a signal is to rotate the Wigner distribution by an angle $\phi=a \pi / 2[7,55,56]$ :

$$
W_{f_{a}}(u, \mu)=W_{f}(u \cos \phi-\mu \sin \phi, u \sin \phi+\mu \cos \phi) .
$$

The Radon transform operator $\mathcal{R} \mathcal{D} \mathcal{N}_{\phi}$, which takes the integral projection of the Wigner distribution of $f(u)$ onto an axis making an angle $\phi$ with the $u$ axis, can be used to restate this property in the following manner [5]:

$$
\left\{\mathcal{R D N}_{\phi}\left[W_{f}(u, \mu)\right]\right\}\left(u_{a}\right)=\left|f_{a}\left(u_{a}\right)\right|^{2},
$$

where $u_{a}$ denotes the axis making angle $\phi=a \pi / 2$ with the $u$ axis. That is, projection of the Wigner distribution of $f(u)$ onto the $u_{a}$ axis gives $\left|f_{a}\left(u_{a}\right)\right|^{2}$, the squared magnitude of the $a$ th order FRT of the function. Hence, the projection axis $u_{a}$ can be referred to as the $a$ th-order fractional Fourier domain (see Fig. 7.1) [7, 55]. The space and frequency domains are merely special cases of the continuum of fractional Fourier domains.

Fig. 7.1 The $a$ th-order fractional Fourier domain [34]

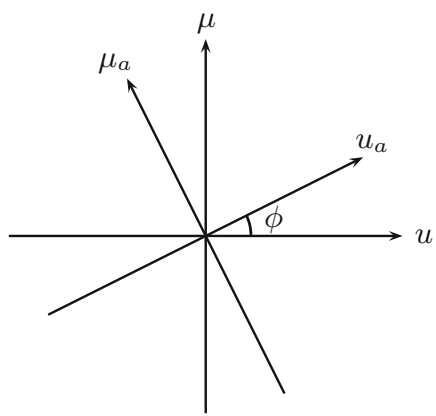


Fractional Fourier domains are recognized as oblique axes in the space-frequency plane [5-7]. By analogy with this concept, the term linear canonical domain has been used in several papers to refer to the domain of the LCT representation of a signal [8-16]. However, it is not immediately obvious from these works where these LCT domains exist and how they are related to the space-frequency plane; in other words, while the effect of an LCT on the space-frequency representation of a signal is well understood as a linear geometrical distortion, it is not immediate how members of the three-parameter family of LCT domains are related to the spacefrequency plane, or how we should visualize them. LCTs are characterized by three independent parameters, and hence LCT domains populate a three-parameter space, which makes them hard to visualize. Below, we explicitly relate LCT domains to the space-frequency plane $[33,34]$. We show that each LCT domain corresponds to a scaled FRT domain, and thus to a scaled oblique axis in the space-frequency plane. Based on this many-to-one association of LCTs with FRTs, LCT domains can be labeled and monotonically ordered by an associated fractional order parameter, instead of their usual three parameters which do not directly lend to a natural ordering.

We use the Iwasawa decomposition to relate the members of the three-parameter family of LCT domains to the space-frequency plane. As given in (7.9), any arbitrary LCT can be expressed as a chirp multiplied and scaled FRT. Thus, in order to compute the LCT of a signal, we can first compute the $a$ th-order FRT of the signal, which transforms the signal to the ath-order fractional Fourier domain. Secondly, we scale the transformed signal. Because scaling is a relatively trivial operation, we need not interpret it as changing the domain of the signal, but merely a scaling of the coordinate axis in the same domain. Finally, we multiply the resulting signal with a chirp to obtain the LCT. Multiplication with a function is not considered an operation which transforms a signal to another domain, but which alters the signal in the same domain. (For instance, when we multiply the Fourier transform of a function with a mask, the result is considered to remain in the frequency domain.) Therefore, only the FRT part of the LCT operation corresponds to a genuine domain change, and the linear canonical transformed signal essentially lives in a scaled fractional Fourier domain. In other words, LCT domains are essentially equivalent to scaled fractional Fourier domains. That is, despite their three parameters, LCT domains do not constitute a richer family of domains than FRT domains. By using the well-known relationship of FRT domains to the space-frequency plane, we can state a similar relationship for the LCT domains as follows:

In the space-frequency plane, the LCT domain $u_{\mathbf{T}}$ with parameter matrix $\mathbf{T}=\left[\begin{array}{lll}A B ; & D\end{array}\right]$ corresponds to a scaled oblique axis making angle $\arctan (B / A)$ with the $u$ axis (or equivalently, having slope $B / A$ ), and scaled with the parameter $M$ where $M=\sqrt{A^{2}+B^{2}}[33,34]$.

Note that any LCT domain is completely characterized by the two parameters $A$ and $B$ (or equivalently by $a$ and $M$, or by $\gamma$ and $\beta$ ) instead of all three of its parameters.

We also note that the relation in (7.17) can be rewritten for the LCT of a signal as

$$
\frac{1}{M}\left\{\mathcal{R D N}_{\phi}\left[W_{f}(u, \mu)\right]\right\}\left(\frac{u}{M}\right)=\left|f_{\mathbf{T}}(u)\right|^{2},
$$


by using (7.17) and (7.9) (with $s=1$ ). This is another way of interpreting scaled oblique axes in the space-frequency plane as the LCT domain with parameter $\mathbf{T}$.

\subsubsection{Essentially Equivalent Domains}

Observe that LCTs with the same value of $B / A$ (or equivalently the same value of $\gamma$ ) will have the same value of $a$ in the decomposition in (7.10), and therefore will be associated with the same FRT domain. We refer to such LCT domains as well as their associated FRT domain as essentially equivalent domains [33, 34]. Note that if a signal has a compact support in a certain LCT domain, then the signal will also have compact support in all essentially equivalent domains.

The concept of essentially equivalent domains we introduce allows many earlier observations and results to be seen in a new light, making them almost obvious or more transparent. For instance, it has been stated that if a particular LCT of a signal is bandlimited, then another LCT of the signal cannot be bandlimited unless $B_{1} / A_{1}=B_{2} / A_{2}$ [10]. Since we recognize the domains associated with two LCTs satisfying this relation to be essentially equivalent, this result becomes obvious. Although we will not further elaborate, other results regarding the compactness/bandlimitedness of different LCTs of a signal [57] can be likewise easily understood in terms of the concept of essentially equivalent domains. As a final example, we consider the LCT sampling theorem, according to which if the LCT of a signal has finite extent $\Delta u_{\mathbf{T}}$, then we should sample it with spacing $\Delta u \leq$ $|B| / \Delta u_{\mathrm{T}}$. Such a sampling scheme collapses when $B=0$. It is easy to understand why if we note that $B=0$ implies that the LCT domain in question is essentially equivalent to the $a=0$ th FRT domain; that is, the domain in which the signal is specified to have finite extent is essentially equivalent to the domain in which we are attempting to sample the signal.

\subsubsection{Optical Interpretation}

Let us now optically interpret the equivalence of LCT domains to FRT domains. Consider a signal that passes through an arbitrary quadratic-phase system. Since the light field at any plane within the system is related to the input field through an LCT, the signal will incrementally be transformed through different LCT domains. Because the three parameters of the consequential LCT domains are not sequenced, it is not easy to give any interpretation or visualize the nature of the transformation of the optical field. However, if we think of the LCT domains as being equivalent to scaled FRT domains, it becomes possible to interpret every location along the propagation axis as an FRT domain of specific order, which is equivalent to an 
oblique axis in the space-frequency plane. Moreover, it has been shown that if we take the fractional order $a$ to be equal to zero at the input of the system, then $a$ monotonically increases as a function of the distance along the optical axis $[41,58]$. In other words, propagation through a quadratic-phase system can be understood as passage through a continuum of scaled FRT domains of monotonically increasing order, instead of passage through an unsequenced plethora of LCT domains [34].

To see that the FRT parameter $a$ is monotonically increasing along the $z$ axis, observe from Eq. (7.10) that $a \propto \arctan (B / A)$, so that $a$ increases with $B / A$. Passage through a lens involves multiplication with the matrix given in Eq. (7.5) which does not change $B / A$. Passage through an incremental section of free space involves multiplication with the matrix given in Eq. (7.6) which always results in a positive increment in $a$. This is because $r$ is proportional to the distance of propagation, and the derivative of the new value of $B / A$ with respect to $r$ is always positive, which implies that $B / A$ always increases with $r$. A similar argument is possible for quadratic graded-index media. A more precise development may be found in $[41,58]$.

Therefore, the distribution of light is continually fractional Fourier transformed through scaled fractional Fourier domains of increasing order, which we know are oblique axes in the space-frequency plane. This understanding of quadraticphase systems yields much more insight into the nature of how light is transformed as it propagates through such a system, as opposed to thinking of it in terms of going through a series of unsequenced LCT domains whose whereabouts we cannot visualize. For example, based on this understanding we show in Sect. 7.7 how to explicitly quantify the degrees of freedom of optical systems with apertures and give conditions for lossless transfer.

\subsection{Degrees of Freedom of Signals and the Bicanonical Width Product}

The conventional space-bandwidth product is of fundamental importance in signal processing and information optics because of its interpretation as the number of degrees of freedom of space- and band-limited signals [5, 17-32]. In this section, we discuss the bicanonical width product $(B W P)$, which is the number of degrees of freedom of LCT-limited signals. The bicanonical width product generalizes the space-bandwidth product and often provides a tighter measure of the actual number of degrees of freedom of signals [33-35]. 


\subsubsection{Space-Bandwidth Product: Degrees of Freedom of Space- and Band-Limited Signals}

Consider a family of signals whose members are approximately confined to an interval of length $\Delta u$ in the space domain and to an interval of length $\Delta \mu$ in the frequency domain in the sense that a large percentage of the signal energy is confined to these intervals. The space-bandwidth product $N$ is then defined $[5,28]$ as

$$
N \equiv \Delta u \Delta \mu
$$

and is always greater than or equal to unity because of the uncertainty relation.

The notion of space-bandwidth product, as degrees of freedom of space- and band-limited signals, can be established in a number of different ways. Here we provide two constructions: one based on Fourier sampling theorem, another based on space-frequency analysis.

\subsubsection{Construction Based on Fourier Sampling Theorem}

The conventional space-bandwidth product is the minimum number of samples required to uniquely identify a signal out of all possible signals whose energies are approximately confined to space and frequency intervals of length $\Delta u$ and $\Delta \mu$. This argument is based on the Shannon-Nyquist sampling theorem, which requires that the spacing between samples (in the space domain) not be greater than $\delta u=1 / \Delta \mu$, so that the minimum number of samples over the space extent $\Delta u$ is given by $\Delta u / \delta u=\Delta u \Delta \mu$. Alternatively, if we sample the signal in the frequency domain, the spacing between samples should not be greater than $\delta \mu=1 / \Delta u$, so that the minimum number of samples over the frequency extent $\Delta \mu$ is given by $\Delta \mu / \delta \mu=\Delta u \Delta \mu$. The minimum number of samples needed to fully characterize an approximately space- and band-limited signal can be interpreted as the number of degrees of freedom of the set of signals. This number of samples turns out to be the same whether counted in the space or frequency domain, and is given by the space-bandwidth product.

\subsubsection{Construction Based on Space-Frequency Analysis}

Another line of development involves space-frequency analysis. When the approximate space and frequency extents are specified as above, this amounts to assuming that most of the energy of the signal is confined to a $\Delta u \times \Delta \mu$ rectangular region in the space-frequency plane, perpendicular to the space-frequency axes (Fig. 7.2). In this case, the area of this rectangular region, which gives the number of degrees of freedom, is equal to the space-bandwidth product. 
Fig. 7.2 Rectangular space-frequency support with area equal to the space-bandwidth product $\Delta u \Delta \mu[34]$

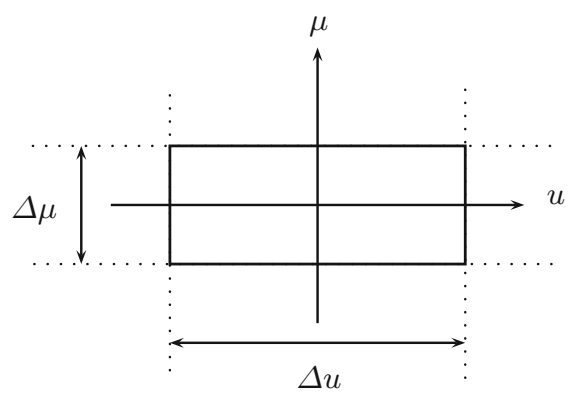

More generally, the number of degrees of freedom is given by the area of the space-frequency support (phase space support), regardless of its shape [5, 30]. When the space-frequency support is not a rectangle perpendicular to the axes, the actual number of degrees of freedom will be smaller than the space-bandwidth product of the signal $[5,30]$.

\subsubsection{Discussion}

The space-bandwidth product is a notion originating from the simultaneous specification of the space and frequency extents. Although this product is commonly seen as an intrinsic property, it is in fact a notion that is specific to the Fourier transform and the frequency domain. It is also possible to specify the extents in other FRT or LCT domains. The set of signals thus specified will in general exhibit a nonrectangular space-frequency support. (For example, we will next show that when two such extents are specified, the support will be a parallelogram $[33,34]$.) In all cases, the area of the support will correspond to the number of degrees of freedom of the set of signals thus defined. If we insist on characterizing this set of signals with conventional space and frequency extents, the space-bandwidth product will overstate the number of degrees of freedom (see Fig. 7.3).

Obviously, specifying a finite extent in a single LCT domain does not define a family of signals with a finite number of degrees of freedom, just as specifying a finite extent in only one of the conventional space or frequency domains does not. However, specifying finite extents in two distinct LCT domains allows us to define a family of signals with a finite number of degrees of freedom. The number of degrees of freedom will depend on both the specified LCT domains and the extents in those domains. 


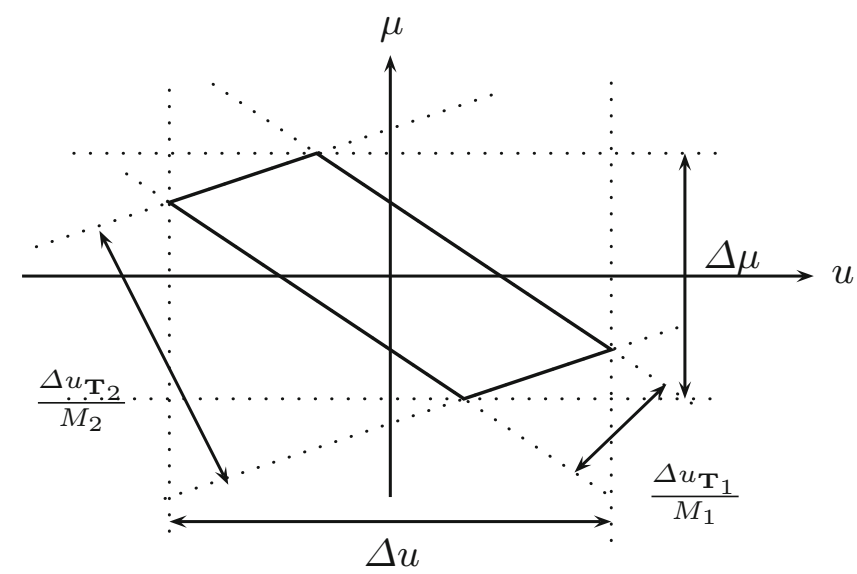

Fig. 7.3 Parallelogram shaped space-frequency support with area equal to the bicanonical width product $\Delta u_{\mathbf{T}_{1}} \Delta u_{\mathbf{T}_{2}}\left|\beta_{1,2}\right|$, which is smaller than the space-bandwidth product $\Delta u \Delta \mu$ [34]

\subsubsection{Bicanonical Width Product: Degrees of Freedom of LCT-Limited Signals}

We first define the space-canonical width product, which gives the number of degrees of freedom of signals that are approximately confined to a finite interval $\Delta u$ in the conventional space domain and to a finite interval $\Delta u_{\mathrm{T}}$ in some other LCT domain [33-35]:

$$
N \equiv \Delta u \Delta u_{\mathbf{T}}|\beta|
$$

This is always greater than or equal to unity because of the uncertainty relation for LCTs $[5,6,10,15]$. Here $\mathbf{T}$ represents the three parameters of the LCT, where $\beta$ is one of these three parameters. The space-canonical width product constitutes a generalization of the space-bandwidth product, and reduces to it when the LCT reduces to an ordinary Fourier transform, upon which $\Delta u_{\mathbf{T}}$ reduces to $\Delta \mu$ and $\beta=1$.

In the above, one of the two domains is chosen to be the conventional space domain. More generally, the two LCT domains can both be arbitrarily chosen. In this case, we use the more general term bicanonical width product $(B W P)$ to refer to the product [33-35]

$$
N \equiv \Delta u_{\mathbf{T}_{1}} \Delta u_{\mathbf{T}_{2}}\left|\beta_{1,2}\right|
$$

where $\Delta u_{\mathbf{T}_{1}}$ and $\Delta u_{\mathbf{T}_{2}}$ are the extents of the signal in two LCT domains and $\beta_{1,2}$ is the parameter of the LCT between these two domains (the LCT which transforms 
the signal from the first LCT domain to the second). Note that the bicanonical width product is defined with respect to two specific LCT domains.

The notion of bicanonical width product as the degrees of freedom of LCTlimited signals can also be established in two ways [34]: based on the LCT sampling theorem, and based on space-frequency analysis. Before establishing this, we note that if $\beta=\infty$ in (7.20) or $\beta_{1,2}=\infty$ in (7.21), then the product $N$ will not be finite and hence the number of degrees of freedom will not be bounded. This is because when this parameter is infinity (that is, $B=0$ ), the two domains are related to each other simply by a scaling or chirp multiplication operation. But as discussed before, domains related by such operations are essentially equivalent. Thus, specification of the extent in two such domains does not constrain the family of signals more than the specification of the extent in only one domain, which, as noted, is not sufficient to make the number of degrees of freedom finite.

\subsubsection{Construction Based on LCT Sampling Theorem}

The space-canonical width product is the minimum number of samples required to uniquely identify a signal out of all possible signals whose energies are approximately confined to a space interval of $\Delta u$ and a particular LCT interval of $\Delta u_{\mathbf{T}}$. (This many number of samples can be used to reconstruct the signal.) This argument can be justified by the use of the LCT sampling theorem. According to the LCT sampling theorem, the space-domain sampling interval for a signal that has finite extent $\Delta u_{\mathbf{T}}$ in a particular LCT domain should not be larger than $\delta u=1 /\left(|\beta| \Delta u_{\mathbf{T}}\right)$. If we sample the space-domain signal at this rate, the total number of samples over the extent $\Delta u$ will be given by $\Delta u / \delta u=\Delta u \Delta u_{\mathbf{T}}|\beta|$, which is precisely equal to the space-canonical width product. Alternatively, if we sample in the LCT-domain, the sampling interval should not be larger than $\delta u_{\mathbf{T}}=1 /(|\beta| \Delta u)$. Sampling at this rate, the total number of samples over the LCT extent $\Delta u_{\mathbf{T}}$ is given by $\Delta u_{\mathbf{T}} / \delta u_{\mathbf{T}}=\Delta u \Delta u_{\mathbf{T}}|\beta|$, which once again is the spacecanonical width product.

The derivation above can be easily replicated for the more general bicanonical width product defined in (7.21). Therefore, the bicanonical width product can also be interpreted as the minimum number of samples required to uniquely identify a signal out of all possible signals whose energies are approximately confined to finite intervals in two specified LCT domains, and therefore as the number of degrees of freedom of this set of signals [33-35].

\subsubsection{Construction Based on Space-Frequency Analysis}

Another line of development involves space-frequency analysis. Here we show that when the extents are specified in two LCT domains as above, the space-frequency support becomes a parallelogram (see Fig. 7.4), and the area of this parallelogram, 
which gives the number of degrees of freedom, is equal to the bicanonical width product [33, 34].

This result follows from the established relationship of LCT domains to the space-frequency plane. Let us consider a set of signals, whose members are approximately confined to the intervals $\left[-\Delta u_{\mathbf{T}_{1}} / 2, \Delta u_{\mathbf{T}_{1}} / 2\right]$ and $\left[-\Delta u_{\mathbf{T}_{2}} / 2, \Delta u_{\mathbf{T}_{2}} / 2\right]$ in two given LCT domains, $u_{\mathbf{T}_{1}}$ and $u_{\mathbf{T}_{2}}$. We want to investigate the space-frequency support of this set of signals. Since LCT domains are equivalent to scaled fractional Fourier domains, each finite interval in an LCT domain will correspond to a scaled interval in the equivalent FRT domain. To see this explicitly, we again refer to (7.9), which implies that if $f_{\mathbf{T}}(u)$ is confined to an interval of length $\Delta u_{\mathbf{T}}$, so is $f_{a}(u / M)$. Therefore, the extent of $f_{a}(u)$ in the equivalent $a$ th-order FRT domain is $\Delta u_{\mathbf{T}} / M$. Thus, the set of signals in question is approximately limited to an extent of $\Delta u_{\mathbf{T}_{1}} / M_{1}$ in the $a_{1}$ th order FRT domain, and an extent of $\Delta u_{\mathbf{T}_{2}} / M_{2}$ in the $a_{2}$ th order FRT domain, where $a_{1}, a_{2}$ and $M_{1}, M_{2}$ are related to $\mathbf{T}_{1}, \mathbf{T}_{2}$ through Eqs. (7.10) and (7.11).

It is well known that if the space-, frequency- or FRT-domain representation of a signal is identically zero (or negligible) outside a certain interval, so is its Wigner distribution [5, 59]. As a direct consequence of this fact, the Wigner distribution of our set of signals is confined to corridors of width $\Delta u_{\mathbf{T}_{1}} / M_{1}$ and $\Delta u_{\mathbf{T}_{2}} / M_{2}$ in the directions orthogonal to the $a_{1}$ th order FRT domain $u_{a_{1}}$, and the $a_{2}$ th order FRT domain $u_{a_{2}}$, respectively. (With the term corridor we are referring to an infinite strip in the space-frequency plane perpendicular to the oblique $u_{a}$ axis. The corridor makes an angle $(a+1) \pi / 2$ with the $u$ axis (see Fig. 7.5).) Now, if we intersect the two corridors defined by each extent, we obtain a parallelogram, which gives the space-frequency support of the signals (see Fig. 7.4). The area of the parallelogram is equal to the bicanonical width product of the set of signals in question. This result will be formally stated as follows:

Consider a set of signals, whose members are approximately confined to finite extents $\Delta u_{\mathbf{T}_{1}}$ and $\Delta u_{\mathbf{T}_{2}}$ in the two LCT domains $u_{\mathbf{T}_{1}}$ and $u_{\mathbf{T}_{2}}$, respectively. Let $\beta_{1,2}$ denote the $\beta$ parameter of the LCT which transforms signals from the first LCT domain to the second. Then, the space-frequency support of these signals is given by a parallelogram defined by these extents (Fig. 7.4), and the area

Fig. 7.4 The space-frequency support when finite extents are specified in two LCT domains. The area of the parallelogram is equal to $\Delta u_{\mathbf{T}_{1}} \Delta u_{\mathbf{T}_{2}}\left|\beta_{1,2}\right|$ [34]

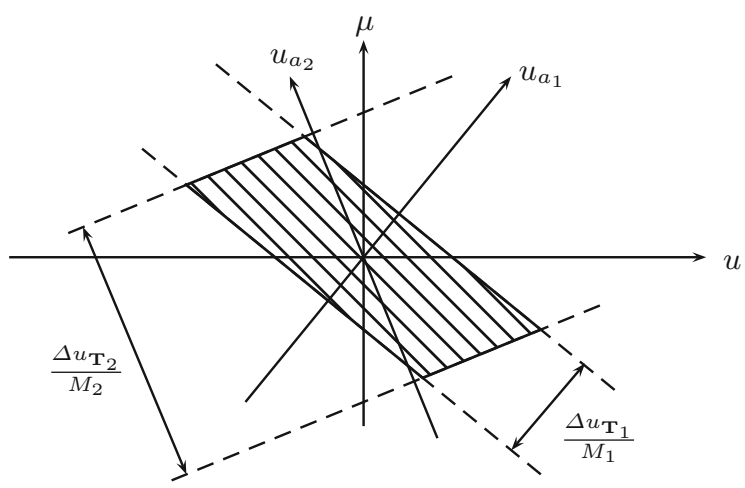


of the parallelogram-shaped support is equal to the bicanonical width product $\Delta u_{\mathbf{T}_{1}} \Delta u_{\mathbf{T}_{2}}\left|\beta_{1,2}\right|$ of the set of signals [33, 34].

Proof. The two heights of the parallelogram defined by the extents $\Delta u_{\mathbf{T}_{1}}$ and $\Delta u_{\mathbf{T}_{2}}$, are $\Delta u_{\mathbf{T}_{1}} / M_{1}$ and $\Delta u_{\mathbf{T}_{2}} / M_{2}$, corresponding to the widths of the corridors. Moreover, the angle between the corridors is $\phi_{2}-\phi_{1}$. Then, the area of the parallelogram is

$$
\begin{aligned}
\text { Area } & =\frac{\Delta u_{\mathbf{T}_{1}}}{M_{1}} \frac{\Delta u_{\mathbf{T}_{2}}}{M_{2}}\left|\csc \left(\phi_{2}-\phi_{1}\right)\right| \\
& =\frac{\Delta u_{\mathbf{T}_{1}} \Delta u_{\mathbf{T}_{2}}}{M_{1} M_{2}\left|\sin \phi_{2} \cos \phi_{1}-\cos \phi_{2} \sin \phi_{1}\right|} \\
& =\frac{\Delta u_{\mathbf{T}_{1}} \Delta u_{\mathbf{T}_{2}}}{\left|A_{1} B_{2}-B_{1} A_{2}\right|} \\
& =\Delta u_{\mathbf{T}_{1}} \Delta u_{\mathbf{T}_{2}} \frac{\left|\beta_{1} \beta_{2}\right|}{\left|\gamma_{1}-\gamma_{2}\right|} \\
& =\Delta u_{\mathbf{T}_{1}} \Delta u_{\mathbf{T}_{2}}\left|\beta_{1,2}\right|,
\end{aligned}
$$

where the third and fourth equality follows from (7.8) and (7.2), respectively. The final result can be obtained from the parameter matrix $\mathbf{T}_{2} \mathbf{T}_{1}^{-1}$ which transforms from the first LCT domain to the second domain.

Since the number of degrees of freedom of a set of signals is given by the area of their space-frequency support, this result provides further justification for interpreting the bicanonical width product as the number of degrees of freedom of LCT-limited signals.

Fig. 7.5 Illustration of a space-frequency corridor [37]

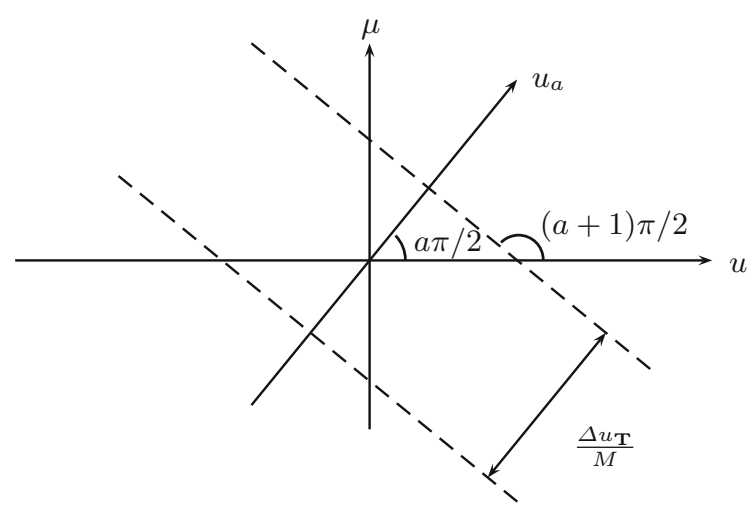




\subsubsection{Discussion}

When confronted with a space-frequency support of arbitrary shape, it is quite common to assume the number of degrees of freedom to be equal to the spacebandwidth product, without regard to the shape of its space-frequency support. In reality, this is a worst-case approach which encloses the arbitrary shape within a rectangle perpendicular to the axes, and overstates the number of degrees of freedom.

The bicanonical width product provides a tighter measure of the number of degrees of freedom than the conventional space-bandwidth product, and allows us to represent and process the signals with a smaller number of samples, since it is possible to enclose the true space-frequency support more tightly with a parallelogram of our choice, as compared to a rectangle perpendicular to the axes, or indeed any rectangle. In applications where the underlying physics involves LCT type integrals (as is the case with many wave propagation problems and optical systems), parallelograms may be excellently, if not perfectly, tailored to the true space-frequency supports of the signals. In the next section, we illustrate how these ideas are useful for representing and reconstructing signals with arbitrary timeor space-frequency support, using fewer samples than required by the ShannonNyquist sampling theorem. The developed approach geometrically amounts to enclosing the support with the smallest possible parallelogram, as opposed to enclosing it with a rectangle as in the classical approach.

Another important feature of the bicanonical width product is that it is invariant under linear canonical transformation. The fact that LCTs model an important family of optical systems, makes the bicanonical width product a suitable invariant measure for the number of degrees of freedom of optical signals. On the other hand, the space-bandwidth product, which is the area of the smallest bounding perpendicular rectangle, may change significantly after linear canonical transformation. This has an important implication in DLCT computation as will be discussed in Sect. 7.6. With this computation method, we can accurately compute an LCT with a minimum number of samples given by the bicanonical width product, so that the bicanonical width product is also a key parameter in fast discrete computation of LCTs, and hence in efficient and accurate simulation of optical systems [35].

Given the fundamental importance of the conventional space-bandwidth product in signal processing and information optics, it is not surprising that the bicanonical width product can also play an important role in these areas. In a later section, we discuss how the bicanonical width product is useful for efficiently and accurately simulating optical systems based on an elegant and natural formulation of DLCT computation. Finally we note that the bicanonical width product has been originally introduced in the context of LCTs $[33,35]$. However, since the equivalence between FRT and LCT domains has been shown [34], we can also speak of the bifractional width product in the context of FRT domains. 


\subsection{Sub-Nyquist Sampling and Reconstruction of Signals}

In this section, we show how to represent and reconstruct signals with arbitrary timeor space-frequency support, using fewer samples than required by the ShannonNyquist sampling theorem [33,36]. The classical Shannon-Nyquist sampling theorem allows us to represent band-limited signals with samples taken at a finite rate. Although the classical approach is optimal for band-limited signals, it is in general suboptimal for representing signals with a known space-frequency support. Application of the classical approach to signals with arbitrarily given spacefrequency support amounts to enclosing the support with a rectangle perpendicular to the space and frequency axes. The number of samples is given by the area of the rectangle and equals the space-bandwidth product, which may be considerably larger than the area of the space-frequency support and hence the actual number of degrees of freedom of the signals. When the space-frequency support is not a rectangle perpendicular to the axes, it is possible to represent and reconstruct the signal with fewer samples than implied by the space-bandwidth product. Light fields propagating through optical systems is one example of an application where nonrectangular supports are commonly encountered [37].

The FRT is a generalization of the Fourier transform and the FRT sampling theorem [49-51] is an extension of the classical sampling theorem (while a special case of the LCT sampling theorem). Based on this generalized sampling theorem, here we provide a sub-Nyquist approach to represent signals with arbitrary spacefrequency support $[33,34,36]$. This approach reduces to the geometrical problem of finding the smallest parallelogram enclosing the space-frequency support. The area of the parallelogram given by the bicanonical width product is the number of samples needed and the reconstruction is given by an explicit formula. This allows us to represent signals with fewer samples than with the classical approach, since it is possible to enclose the true space-frequency support more tightly with a parallelogram of our choice, than with a rectangle perpendicular to the axes. A Wigner-based approach to related problems has been given in $[47,60]$.

\subsubsection{Constrained Signal Representation}

Our goal is to determine the minimal sampling rate when the space-frequency support is given and to show how to reconstruct the signal from those samples. First we consider the (constrained) case where the signal needs to be sampled in a specific domain, say the space domain $u$. In other words, we are not free to choose the domain in which to sample the signal and must sample it in the specified domain. Without loss of generality, suppose the specified domain is the space domain. In the classical approach, the sampling rate in the space domain is determined by the extent in the frequency domain. If we denote this extent by $\Delta \mu$, then the spacing between space-domain samples must not be greater than $\delta u=1 / \Delta \mu$, so that the minimum 
number of samples over the space extent $\Delta u$ is given by $\Delta u /(1 / \Delta \mu)=\Delta u \Delta \mu$, which is the space-bandwidth product. (The space extent $\Delta u$ is the projection of the space-frequency support onto the $u$ axis.) This classical approach is geometrically equivalent to enclosing the support with a rectangle perpendicular to the space and frequency axes and having sides of length $\Delta u$ and $\Delta \mu$. Its area equals $\Delta u \Delta \mu$ and gives the number of samples required for interpolating the continuous signal in the Nyquist-Shannon sense (Fig. 7.6).

For efficient sampling, it is desirable to approach the minimum number of samples possible given by the area of the space-frequency support. When we use the FRT sampling theorem, the sampling rate can be determined by the extent in the FRT domain which minimizes the required number of the samples. Since signals that are extent limited in two FRT domains have parallelogram shaped supports (see Fig. 7.7), determining the optimal value of $a$ is equivalent to the problem of finding the smallest parallelogram enclosing the space-frequency support, under the constraint that two sides of the parallelogram must be perpendicular to the $u$ axis (Fig. 7.6). (This constraint arises because the signal must be sampled specifically in the space domain.) The minimum number of samples needed for reconstruction (based on the FRT sampling theorem) is given by the area of this enclosing parallelogram, which is equal to the bicanonical width product for the two FRT domains orthogonal to the sides of the parallelogram. Reconstruction of the continuous signal is possible through the interpolation formula associated with the FRT sampling theorem, which is a special case of the LCT interpolation formula in $(7.15)[33,34,36]$.

This approach is illustrated in Fig. 7.6, where the shaded region shows the spacefrequency support. In the classical approach, we would be finding the smallest rectangle perpendicular to the axes that encloses the space-frequency support of the signal. With the proposed approach, we find the smallest enclosing parallelogram with two sides perpendicular to the space axis. Since the FRT includes the ordinary Fourier transform as a special case (and parallelograms include rectangles), the proposed approach will never require more samples than the classical approach. On the other hand, the freedom to optimally choose $a$ can result in a fewer number of samples being necessary $[33,34,36]$. (That is, the area of the fitting parallelogram will be always less than or equal to the area of the fitting rectangle.)

Fig. 7.6 The smallest enclosing parallelogram (solid) and rectangle (dashed), both under the constraint that two sides be perpendicular to the space axis $u$. The shaded region is the space-frequency support $[33,36]$

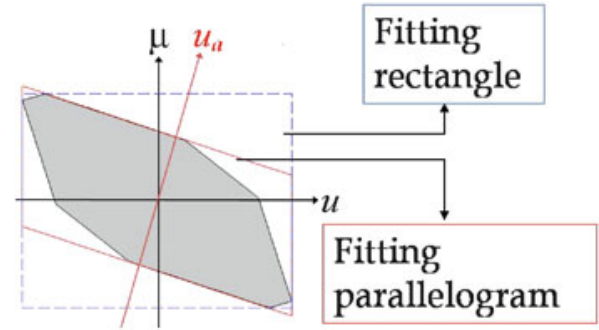


Fig. 7.7 The space-frequency support of $f(u)($ left $)$ and $f_{\mathbf{T}}(u)(r i g h t)$ for space- and LCT-limited signals. The area of both parallelograms are equal to $\Delta u \Delta u_{\mathbf{T}}|\beta|[34]$
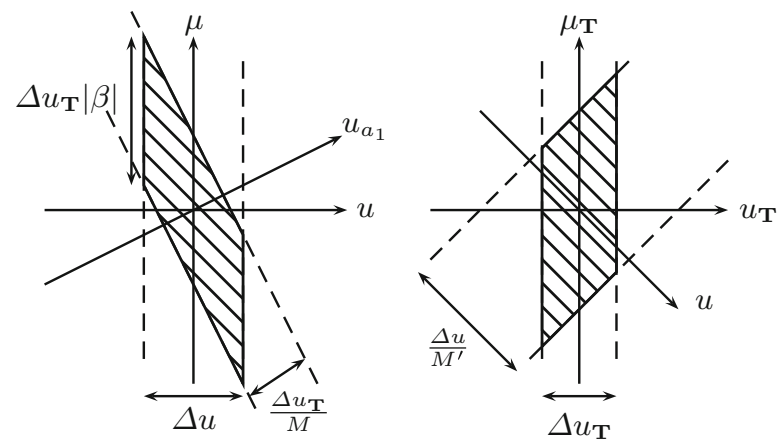

\subsubsection{Unconstrained Signal Representation}

In some applications we may have the freedom to process the analog signal prior to sampling and hence to sample the signal at a domain of our choice. In this case the number of samples can be further reduced [33]. This involves computing the FRT of the analog signal prior to sampling. Such computations may involve chirp modulators for time-domain signals and lenses for space-domain signals [5]. After sampling, if necessary we can return back to the original domain in $\sim N \log N$ time since discrete FRTs can be computed in this amount of time [35].

The FRT sampling theorem allows us to work with any two arbitrary domains (the sampling domain and the domain where the extent determines the sampling rate) since any such domains can be related through the FRT; hence, we are free to determine the sampling rate from the extent in any FRT domain of our choice. This allows us to further reduce the number of samples by enclosing the support with an arbitrary parallelogram, instead of a rectangle. This approach reduces to a simple geometrical problem which requires us to find the minimum-area parallelogram enclosing the given space-frequency support. In contrast to the constrained case, having the flexibility of sampling in any FRT domain removes the requirement that the two sides of the parallelogram be perpendicular to the space domain, and allows us to fit an arbitrary parallelogram. The number of samples required is given by the area of the parallelogram, which is equal to the bicanonical width product. The signal can be represented optimally through its samples at either of the two FRT domains that are orthogonal to the sides of the best-fitting parallelogram. Note that this approach gives us two optimal FRT domains in which the signal should be sampled. It is also possible to represent the signal in any essentially equivalent LCT domain, with the same sampling efficiency $[33,34]$. 


\subsection{Efficient Discrete LCT Computation for System Simulation}

We now review how the bicanonical width product is useful for the efficient and accurate simulation of optical systems, based on a natural formulation of discrete LCT (DLCT) computation [35, 61]. It has been recently shown that if the number of samples $N$ is chosen to be at least equal to the bicanonical width product, the DLCT can be used to obtain a good approximation to the continuous LCT, limited only by the fundamental fact that a signal cannot have strictly finite extent in more than one domain $[35,61]$. The exact relation between the discrete and continuous LCT precisely shows the approximation involved and demonstrates how the approximation improves with increasing $N$ [35]. Because this exact relation generalizes the corresponding relation for Fourier transforms [62], the DLCT defined in [63] approximates the continuous LCT in the same sense that the DFT approximates the continuous Fourier transform, provided the number of samples and the sampling intervals are chosen based on the LCT sampling theorem as specified in $[35,61]$.

We also note that this DLCT can be efficiently computed in $\mathcal{O}(N \log N)$ time by successively performing a chirp multiplication, a fast Fourier transform (FFT), and a second chirp multiplication, by taking advantage of the simple form of the DLCT $[35,52,63]$. This straightforward fast computation approach does not require sophisticated algorithms or space-frequency support tracking for accurately computing the continuous LCT, as opposed to other LCT computation methods [64-69]. To summarize, a simple fast computation method, a well-defined relationship to the continuous LCT, and unitarity make this definition of the DLCT an important candidate for being a widely accepted definition of the discrete version of the LCT [35].

Note that in order to use any DLCT definition in practice, to approximately compute the samples of the LCT of a continuous signal, it is necessary to know how to choose the number of samples and the sampling intervals, based on some prior information about the signal. The described computation approach (first discussed in [61], and then independently developed in [35]) meets precisely this demand and allows us to accurately compute LCTs with the minimum possible number of samples. In this formulation, the extents of the signal in the input and output LCT domains (the original space domain and the target LCT domain) are assumed to be specified as prior information. This is equivalent to assuming an initial parallelogram-shaped space-frequency support [33, 34, 70]. The minimum number of samples required for accurate computation is then determined from the LCT sampling theorem. This minimum number of samples is equal to the bicanonical width product, which is also the area of the parallelogram support $[33,35]$. The DLCT defined in [63] works with this minimum number of samples without requiring any oversampling at the intermediate stages of the computation, in contrast to previously given approaches $[66,67]$ for the same DLCT. On the other hand, use of the Shannon-Nyquist sampling theorem instead of the LCT sampling theorem, as 
in $[66,67]$, leads to problems such as the need to use a greater number of samples, different sampling rates at intermediate stages of the computation, or different numbers of samples at the input and output domains.

This natural DLCT computation method has been revisited in [70], where an interpretation of the method has been given through phase-space diagrams. This allows us to see from yet another perspective how this elegant and accurate LCT computation method $[35,61]$ works with the minimum number of samples, without requiring interpolation. The DLCT computation presented in $[35,61]$ and the phasespace illustrations in $[70,71]$ assume that the extent of the signal is known at the input and output of the system to be simulated. We now discuss how to optimally simulate optical systems by using this DLCT computation method when the spacefrequency support of the input signal is specified [36] (rather than its extents in the input and output domains). Different assumptions about the initial space-frequency support have been made in the literature to explore efficient DLCT computation $[65-69,72,73]$. Hence here we explore a unified method that works with any initial support while still ensuring the minimality of the number of samples [36]. The idea is to find the number of samples by fitting a parallelogram to the given space-frequency support, such that two opposing sides are perpendicular to the $u$ axis (the input domain) and the other sides are perpendicular to the oblique axis corresponding to the output LCT domain. The area of the smallest fitting parallelogram gives the number of samples that needs to be used for an accurate DLCT computation [36]. Then the samples of the continuous signal at the output of the optical system can be obtained by sampling the input signal at this rate and then computing its DLCT as described in [35].

This elegant DLCT formulation is mainly achieved through the property that the bicanonical width product is an invariant measure for the number of degrees of freedom of signals under linear canonical transformation [33, 34]. To see this, suppose a finite extent has been specified in the space domain and in some other LCT domain. The corresponding space-frequency support is shown in Fig. 7.7a. If we transform to precisely the same LCT domain in which the extent has been specified, the new space-frequency support becomes as shown in Fig. 7.7b. Here $M$ and $M^{\prime}$ are the scaling parameters associated with the LCT and inverse LCT operations, respectively. Note that in both parts of the figure, the support is bounded by a vertical corridor, perpendicular to the space domain in part a, and to the LCT domain in part b. We are not surprised that the transformed support is again a parallelogram, since the linear geometric distortion imparted by an LCT always maps a parallelogram to another parallelogram. Moreover, the areas of both parallelograms are equal to each other and given by the bicanonical width product $\Delta u \Delta u_{\mathbf{T}}|\beta|$, so that the number of degrees of freedom as measured by the bicanonical width product remains the same after the LCT. This does not surprise us either, since LCTs are known not to change the support area in phase space. The fact that LCTs model an important family of optical systems makes the bicanonical width product a suitable invariant measure for the number of degrees of freedom of optical signals. 
On the other hand, the space-bandwidth product, which is the area of the smallest bounding perpendicular rectangle, may change significantly after linear canonical transformation, and quadratic-phase optical systems. (This is the reason why the number of samples must be increased at some intermediate stages of certain previously proposed FRT and LCT algorithms which rely on the space-bandwidth product either as the measure of the number of degrees of freedom or as the minimum number of samples required [65-69, 72, 73]. In contrast, fast computation of LCTs based on the results presented in [35, 61] allows us to work with the same number of samples in both domains without requiring any oversampling. This number of samples is the minimum possible for both domains based on the LCT sampling theorem, and is given by the bicanonical width product [35].) This factor makes the conventional space-bandwidth product undesirable as a measure of the number of degrees of freedom, which we expect to be an intrinsic and conserved quantity under invertible unitary transformations.

The so-called generalized space-bandwidth product, which essentially removes the requirement that the rectangular support be perpendicular to the axes, has been proposed [74] as an improvement over the conventional space-bandwidth product. A related approach has also been studied [60]. It has been noted that this entity is invariant under the FRT operation (rotational invariance), but it has also been emphasized that "further research is required in obtaining other forms of generalized space-bandwidth products that are invariant under a more general area preserving space-frequency operations: the symplectic transforms" [74]. The bicanonical width product meets precisely this demand and allows us to compute LCTs with the minimum possible number of samples without requiring any interpolation or oversampling at intermediate stages of the computation [35].

\subsection{Degrees of Freedom of Optical Systems}

We now discuss how to explicitly quantify the degrees of freedom of first-order optical systems with multiple apertures, and give explicit conditions for lossless transfer $[33,37]$. In particular, we answer the following questions about apertured optical systems, which here refers to systems consisting of an arbitrary sequence of thin lenses and apertures separated by sections of free space:

- Given the space-frequency support of an input signal and the parameters of an apertured optical system, will there be any information loss upon passage through the system?

- Which set of signals can pass through a given apertured system without any information loss? In other words, what is the largest space-frequency support that can pass through the system without any information loss?

- What is the maximum number of spatial degrees of freedom that can be supported by a given apertured system? 
The space-frequency support (phase-space support) of a set of signals may be defined as the region in the space-frequency plane (phase space) in which a large percentage of the total energy is confined [5,30]. The number of degrees of freedom is given by the area of the space-frequency support. We also define the space-frequency window (phase-space window) of a system [33, 37] as the largest space-frequency support that can pass through the system without any information loss. Here we develop a simple method to find the space-frequency window of a given system in terms of its parameters. Once the space-frequency window of the system is determined, it specifies the set of all signals that can pass through the system without information loss: the optical system preserves the information content of signals whose space-frequency supports lie inside the system window. All we need to do is to compare the space-frequency support of the input signal with the space-frequency window of the system. If the signal support lies completely inside the system window, the signal will pass through the system without any information loss. Otherwise, information loss will occur.

The number of degrees of freedom of the set of signals which can pass through a system can be determined from the area of the space-frequency window of the system. Although the space-frequency window may in general have different shapes $[5,30]$, it is often assumed to be of rectangular shape with the spatial extent determined by a spatial aperture in the object or image plane, and the frequency extent determined by an aperture in a Fourier plane. (Again, we consider onedimensional signals and systems for simplicity.) If these apertures are of length $\Delta x$ and $\Delta \sigma_{x}$ respectively, then the number of degrees of freedom that can be supported by the system is given by $\Delta x \Delta \sigma_{x}$. More generally, for space-frequency windows of different (non-rectangular) shapes, the number of degrees of freedom is given by the area of the space-frequency window [33, 37].

Physical systems which carry or process signals always limit their spatial extents and bandwidths to certain finite values. A physical system cannot allow the existence of frequencies outside a certain band because there is always some limit to the resolution that can be supported. Likewise, since all physical events of interest have a beginning and an end, or since all physical systems have a finite extent, the temporal duration or spatial extent of the signals will also be finite. For example, in an optical system the sizes of the lenses will limit both the spatial extent of the images that can be dealt with and their spatial bandwidths. More generally, we may say that they will limit the signal to a certain region in the space-frequency plane. We refer to this region as the space-frequency window of the system. It is these physical limitations that determine the space-frequency support of the signals and thus their degrees of freedom. Just as these may be undesirable physical limitations which limit the performance of the system, they may also be deliberate limitations with the purpose of limiting the set of signals we are dealing with. When a signal previously represented by a system with larger space-frequency window is input into a system with smaller space-frequency window, information loss takes place.

The conventional space-bandwidth product has been of fundamental importance because of its interpretation as the number of degrees of freedom [5, 17-23, 25-28, $30-32,75,76]$. In most works, the space-bandwidth product, as its name implies, is 
the product of a spatial extent and a spatial-frequency extent. This implies the assumption of a rectangular space-frequency region. However, the set of input signals may not exhibit a rectangular space-frequency support, and even if they do, this support will not remain rectangular as it propagates through the system [33-35]. Likewise, the space-frequency windows of multi-component optical systems, as we will see in this section, do not in general exhibit rectangular shapes. This possibility and some of its implications were discussed in [30]. In [33, 37] we made concrete the hypothetical concept of a non-rectangular space-frequency window, and showed how it can be actually computed for a broad class of optical systems, as will be discussed here. (To prevent possible confusion, we underline that we are dealing with systems with sequentially cascaded apertures, and not systems with multiple parallel apertures.)

We also note that the phase-space window has been referred to by different names, such as the space-bandwidth product of the system (in short SWY) $[30,77,78]$, the system transmission range [77], and the Wigner or space-bandwidth chart of the system $[77,78]$. Also, the concept of degrees of freedom can be related to other concepts such as Shannon number and information capacity of an optical system [76], geometrical etendue [79], dimensionality, and so on.

In order to treat systems with real physical parameters, we first revisit some of the background material discussed in Sect. 7.2, and translate them to their dimensional counterparts. We then discuss how to find the phase-space window of an optical system. Next, we treat the cases of lossless and lossy transfer separately, and finally conclude with a discussion of applications.

\subsubsection{Scale Parameters and Dimensions}

Dimensionless variables and parameters were employed in the previous sections for simplicity and purity (see Sect. 7.2). In this section, we will employ variables with real physical dimensions. For this, we need to revisit a number of earlier definitions and results. When dealing with FRTs, the choice of scale and dimensions must always be noted, as this has an effect on the fractional order observed at a given plane in the system [5, pp. 320-321]. Using $x$ to denote a dimensional variable (with units of length), the ath-order FRT [5] of a function $\hat{f}(x)$, denoted by $\hat{f}_{a}(x)$, can be defined as

$$
\begin{aligned}
\hat{f}_{a}(x) & \equiv\left(\hat{\mathcal{F}}^{a} \hat{f}\right)(x) \equiv \int_{-\infty}^{\infty} \hat{K}_{a}\left(x, x^{\prime}\right) \hat{f}\left(x^{\prime}\right) d x^{\prime}, \\
\hat{K}_{a}\left(x, x^{\prime}\right) & \equiv \frac{A_{\phi}}{s} e^{i \pi\left(\frac{\cot \phi}{s^{2}} x^{2}-2 \frac{\csc \phi}{s^{2}} x x^{\prime}+\frac{\cot \phi}{s^{2}} x^{\prime 2}\right)} .
\end{aligned}
$$

Here $s$ is an arbitrary scale parameter with dimensions of length. The scale parameter $s$ serves to convert the dimensional variables $x$ and $x^{\prime}$ inside the FRT integral to dimensionless form. A hat over a function or kernel shows that it takes 
dimensional arguments [5, pp. 224-227]. The FRT definition above reduces to the pure mathematical FRT definition with dimensionless arguments if we define the dimensionless variables $u=x / s$ and $u^{\prime}=x^{\prime} / s$, or simply if we set $s=1$ in our measurement unit (meters, etc.). The choice $s=1$ unit makes the expressions simpler, but we feel that this merely hides the essential distinction between dimensional and dimensionless variables and would actually be a disservice to the reader.

We will denote the LCT of a function $\hat{f}(x)$ with the dimensional parameter matrix $\hat{\mathbf{T}}=[\hat{A} \hat{B} ; \hat{C} \hat{D}]$ as $\hat{f}_{\hat{\mathbf{T}}}(x)$ :

$$
\begin{aligned}
\hat{f}_{\hat{\mathbf{T}}}(x) & \equiv\left(\hat{\mathcal{C}}_{\hat{\mathbf{T}}} \hat{f}\right)(x) \equiv \int_{-\infty}^{\infty} \hat{C}_{\hat{\mathbf{T}}}\left(x, x^{\prime}\right) \hat{f}\left(x^{\prime}\right) d x^{\prime}, \\
\hat{C}_{\hat{\mathbf{T}}}\left(x, x^{\prime}\right) & \equiv \sqrt{\frac{1}{\hat{B}}} e^{-i \pi / 4} e^{i \pi\left(\frac{\hat{D}}{\hat{B}} x^{2}-2 \frac{1}{\hat{B}} x x^{\prime}+\frac{\hat{A}}{\hat{B}} x^{\prime 2}\right)},
\end{aligned}
$$

for $\hat{B} \neq 0$. A hat over a parameter shows that it is the dimensional counterpart of the same parameter without the hat. Any LCT can be decomposed into a (dimensional) FRT followed by scaling followed by chirp multiplication [5, 37]:

$$
\hat{\mathbf{T}}=\left[\begin{array}{cc}
\hat{A} & \hat{B} \\
\hat{C} & \hat{D}
\end{array}\right]=\left[\begin{array}{cc}
1 & 0 \\
-\frac{q}{s^{2}} & 1
\end{array}\right]\left[\begin{array}{cc}
M & 0 \\
0 & \frac{1}{M}
\end{array}\right]\left[\begin{array}{cc}
\cos \phi & s^{2} \sin \phi \\
-\frac{\sin \phi}{s^{2}} & \cos \phi
\end{array}\right] .
$$

The three matrices, respectively, correspond to the transformation matrices of chirp multiplication with parameter $q$ (multiplication by exp $\left(-i \pi \frac{q}{s^{2}} x^{2}\right)$ ), coordinate scaling with factor $M>0$ (mapping of $\hat{f}(x)$ into $\sqrt{1 / M} \hat{f}(x / M)$ ), and ath order dimensional FRT with $\phi=a \pi / 2$ (transformation of $\hat{f}(x)$ into $\hat{f}_{a}(x)$ ). The decomposition can be written more explicitly in terms of the LCT and FRT domain representations of the signal $\hat{f}(x)$ as

$$
\hat{f}_{\hat{\mathbf{T}}}(x)=\exp \left(-i \pi \frac{q}{s^{2}} x^{2}\right) \sqrt{\frac{1}{M}} \hat{f}_{a}\left(\frac{x}{M}\right) .
$$

This is the dimensional version of the Iwasawa decomposition in (7.9).

By appropriately choosing the three parameters $a, M, q$, the above equality can be satisfied for any $\hat{\mathbf{T}}=[\hat{A} \hat{B} ; \hat{C} \hat{D}]$ matrix. Solving for $a, M, q$ in (7.8), we obtain the decomposition parameters in terms of the matrix entries $\hat{A}, \hat{B}, \hat{C}, \hat{D}$ :

$$
\begin{gathered}
a= \begin{cases}\frac{2}{\pi} \arctan \left(\frac{1}{s^{2}} \frac{\hat{B}}{\hat{A}}\right), & \text { if } \hat{A} \geq 0 \\
\frac{2}{\pi} \arctan \left(\frac{1}{s^{2}} \frac{\hat{B}}{\hat{A}}\right)+2, & \text { if } \hat{A}<0\end{cases} \\
M=\sqrt{\hat{A}^{2}+\left(\hat{B} / s^{2}\right)^{2}},
\end{gathered}
$$




$$
q= \begin{cases}-s^{2} \frac{\hat{C}}{\hat{A}}-\frac{1}{s^{2}} \frac{\hat{B} / \hat{A}}{\hat{A}^{2}+\left(\hat{B} / s^{2}\right)^{2}}, & \text { if } \hat{A} \neq 0 \\ -s^{2} \frac{\hat{D}}{\hat{B}}, & \text { if } \hat{A}=0\end{cases}
$$

The range of the arctangent lies in $(-\pi / 2, \pi / 2]$.

\subsubsection{Phase-Space Window of Optical Systems}

We now describe how to find the phase-space window (space-frequency window) of an apertured optical system $[33,37]$. Such systems consist of arbitrary concatenations of apertures with quadratic-phase systems (which in turn consist of an arbitrary number of lenses, sections of free space and quadratic graded-index media). Also note that a lens with a finite aperture can be viewed as an ideal lens followed by a finite aperture. Although beyond the scope of the present discussion, these results can be extended to more general systems involving occlusions [80], prisms and gratings [78], and bends and shifts of the optical axis.

Let us first introduce the notation. The input and output planes are defined along the optical axis $z$ at $z=0$ and $z=d$, where $d$ is the length of the system. If the apertures did not exist, the amplitude distribution at any plane perpendicular to the optical axis could be expressed as an LCT of the input. Hence each $z$ plane corresponds to an LCT domain. Let $L$ denote the total number of apertures in the system. $z_{j}$ and $\Delta_{j}$ denote the location and extent of the $j$ th aperture in the system, where $j=1,2, \ldots, L$. The matrix $\hat{\mathbf{T}}_{j}$ is used to denote the parameter matrix of the system from the input to the position of the $j$ th aperture; that is, the system lying between 0 and $z_{j}$ excluding the apertures. The matrix $\hat{\mathbf{T}}_{j}$ can be readily calculated using the matrices for lenses, sections of free space, quadratic graded-index media, and the concatenation property [5]. The matrix elements of $\hat{\mathbf{T}}_{j}$ is denoted by $\hat{A}_{j}, \hat{B}_{j}$, $\hat{C}_{j}, \hat{D}_{j}$. The associated Iwasawa decomposition parameters is denoted by $a_{j}, M_{j}, q_{j}$, which can be computed from $\hat{A}_{j}, \hat{B}_{j}, \hat{C}_{j}, \hat{D}_{j}$ by using the formulas (7.31), (7.32), (7.33). The FRT order in the Iwasawa decomposition begins from 0 at the input of the system, and then monotonically increases as a function of distance [5, 34].

For lossless transfer through the system, the extent of the signal just before each aperture must lie inside the aperture. For simplicity we assume that both the aperture and the signal extents are centered around the origin. Then, the following must be satisfied for $j=1,2, \ldots, L$ :

$$
\Delta x_{\hat{\mathbf{T}}_{j}} \leq \Delta_{j}
$$

where $\Delta x_{\hat{\mathbf{T}}_{j}}$ denotes the extent of the signal in the $x_{\hat{\mathbf{T}}_{j}}$ domain, which corresponds to the LCT domain at the $z=z_{j}$ plane, where the $j$ th aperture is situated.

As we have showed before, LCT domains are equivalent to scaled FRT domains and thus to scaled oblique axes in the space-frequency plane [34]. Based on this 
equivalence, each finite interval in an LCT domain will correspond to a scaled interval in the equivalent FRT domain. To see this explicitly in the dimensional case, we refer to (7.30), which implies that if the linear canonical transformed signal $\hat{f}_{\hat{\mathbf{T}}}(x)$ is confined to an interval of length $\Delta x_{\hat{\mathbf{T}}}$, so is $\hat{f}_{a}(x / M)$. Therefore, the extent of the fractional Fourier transformed signal $\hat{f}_{a}(x)$ in the equivalent FRT domain is $\Delta x_{\hat{\mathbf{T}}} / M$. Thus, the condition in (7.34) can be reexpressed as

$$
\Delta x_{a_{j}} \leq \Delta_{j} / M_{j},
$$

where $\Delta x_{a_{j}}$ denotes the extent of the signal in the $a_{j}$ th order (dimensional) FRT domain.

FRT domains are often visualized in the dimensionless space-frequency plane where the coordinates are scaled such that the space and frequency axes are dimensionless. This is achieved by introducing the scaling parameter $s$ and the dimensionless scaled coordinates $u=x / s$ and $\mu=s \sigma_{x}$. The condition for lossless information transfer then becomes

$$
\Delta x_{a_{j}} / s \leq \Delta_{j} / M_{j} s,
$$

where $\Delta x_{a_{j}} / s$ denotes the extent of the signal in the $a_{j}$ th order (dimensionless) FRT domain (along the oblique axis making angle $a_{j} \pi / 2$ with the $u=x / s$ axis). In other words, for every $j=1,2, \ldots, L$, the signal must be confined to the normalized aperture extent of $\Delta_{j} / M_{j} s$ along the oblique axis with angle $a_{j} \pi / 2$.

It is well known that if the space-, frequency-, or FRT-domain representation of a signal is identically zero (or negligible) outside a certain interval, so is its Wigner distribution [5, 59]. As a direct consequence of this fact, the condition in (7.36) defines a corridor of width $\Delta_{j} / M_{j} s$ in the direction orthogonal to the $a_{j}$ th order FRT domain $u_{a_{j}}$. (With the term "corridor" we are referring to an infinite strip in the space-frequency plane perpendicular to the oblique $u_{a_{j}}$ axis.) The corridor makes an angle $\left(a_{j}+1\right) \pi / 2$ with the $u=x / s$ axis in the dimensionless space-frequency plane (see Fig. 7.5). Now, if we intersect the corridors defined by each aperture, we obtain a bounded region in the space-frequency plane, which has the form of a centrally symmetrical convex polygon (see Fig. 7.8 for $L=2$ and Fig. 7.9 for $L=4$ ). We refer to this convex polygon defined by the normalized aperture extents as the space-frequency window of the system [37].

The space-frequency window specifies the set of all signals that can pass through the system without any loss: the optical system preserves the information content of all signals whose supports lie inside the space-frequency window. The area of the space-frequency window gives the number of degrees of freedom that can pass through the system. This is also the minimum number of samples required to faithfully represent an arbitrary signal at the output of the system.

We can summarize the steps for finding the phase-space window (spacefrequency window) as follows [37]: 
1. Compute the parameter matrix $\hat{\mathbf{T}}_{j}$ for each aperture $j=1,2, \ldots, L$ using the matrices for lenses, sections of free space and quadratic graded-index media, and the concatenation property. Recall that $\hat{\mathbf{T}}_{j}$ was defined as the parameter matrix of the system lying between the input plane and the location of the $j$ th aperture.

2. Compute the corresponding Iwasawa decomposition parameters $a_{j}$ and $M_{j}$ (the fractional order and the magnification) by inserting the matrix entries $\hat{A}_{j}, \hat{B}_{j}, \hat{C}_{j}$, $\hat{D}_{j}$ into the formulas (7.31) and (7.32).

3. In the dimensionless space-frequency plane, draw a corridor of width $\Delta_{j} / M_{j} s$ making angle $\left(a_{j}+1\right) \pi / 2$ with the $x / s$ axis, for each $j$ (see Fig. 7.9). The corridor is explicitly defined by the following two lines: $y=-\cot \left(a_{j} \pi / 2\right) x \pm$ $\frac{\Delta_{j}}{2 M_{j} s} \csc \left(a_{j} \pi / 2\right)$.

4. Intersect the corridors from all apertures to determine the region lying inside all the corridors. This is the phase-space window at the input plane $z=0$.

5. Scale the horizontal and vertical coordinates by $s$ and $1 / s$, respectively, to obtain the phase-space window in the dimensional space-frequency plane $x-\sigma_{x}$.

A few remarks are in order at this point. First, the area of the window and hence the number of degrees of freedom of the system remains the same whether

Fig. 7.8 Space-frequency window of a system with two apertures [37]
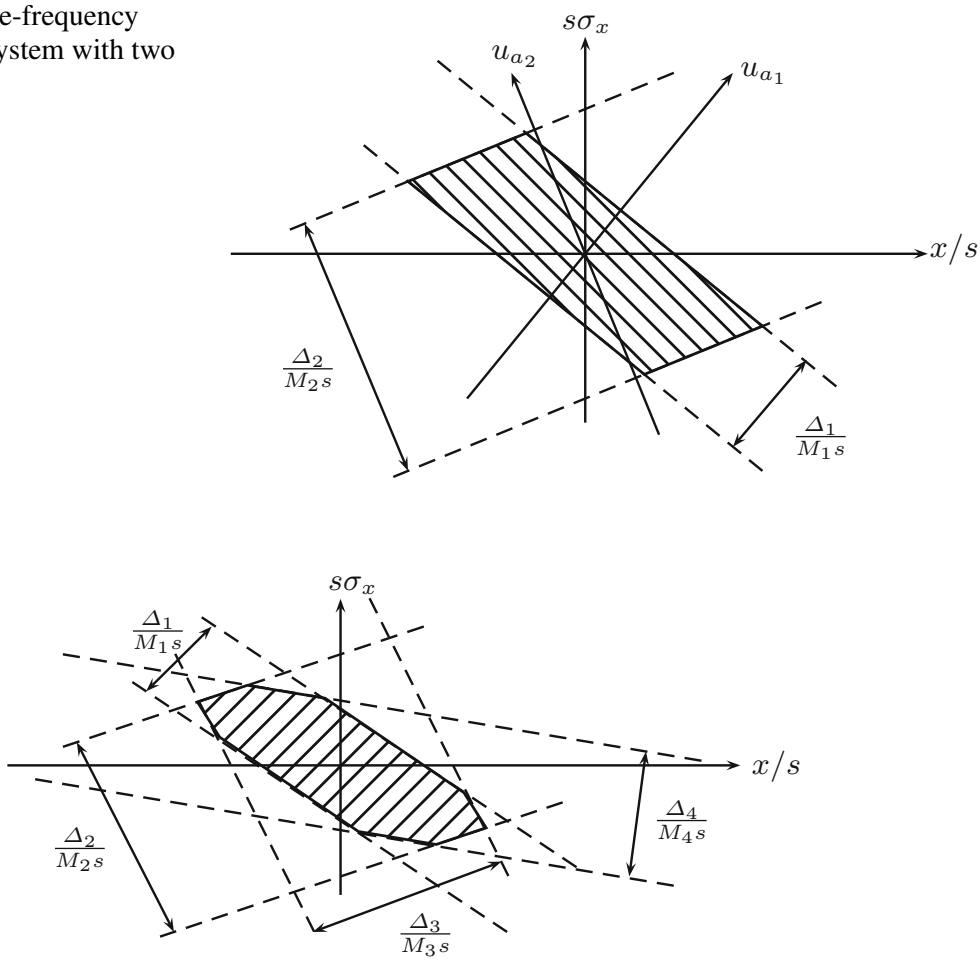

Fig. 7.9 Space-frequency window of a system with four apertures [37] 
it is computed in dimensional or dimensionless space. Second, choice of the scale parameter $s$ is arbitrary and the system window in the dimensional space-frequency plane is independent of the choice of $s$. However, choice of $s$ does affect the value of $a$ as a function of $z$. Some choices better utilize the range of $a$ (as in Fig. 7.10), whereas poor choices lead to $a$ changing too quickly over a short range of $z$ and then saturating [5, pp. 320-321, 377-378]. One approach is to choose $s$ such that the space and frequency extents in the dimensionless space-frequency plane are comparable to each other. Third, the system window is computed with respect to a chosen reference plane. Above, we compute it with respect to the input plane, so that we can directly compare the input signal support with the system window. The phase-space window at the input plane can be shortly referred to as the input phase-space window of the system. If one desires to visualize the system window with respect to a different reference plane, it can be transformed to the new plane using the LCT transformation from the input plane to the new reference plane [37]. (More explicitly, if the $i$ th corner of the system window is expressed as $\left(x^{(i)}, \sigma_{x}^{(i)}\right)$, then after LCT transformation with matrix $\hat{\mathbf{T}}$, the new corner will be described by the coordinates $\hat{\mathbf{T}}\left[x^{(i)} \sigma_{x}^{(i)}\right]^{T}$, where $T$ is the transpose operation $[33,65]$.)

We now illustrate the method on a sample system. Figure $7.10 \mathrm{a}$ shows a system consisting of several apertures and lenses, whose aperture sizes and focal lengths are given right above them. The fractional transform order $a$ and the scale parameter $M$ of the system are plotted in Fig. 7.10 as functions of distance $z$. The emphasis in this paper is on computing $a_{j}$ and $M_{j}$ at the aperture locations, since these allow us to determine the system window. However, these quantities can also be computed for all values of $z$ in the system, revealing their continual evolution as we move along the optical axis, as illustrated in Fig. 7.10b, c. We can compute $a(z)$ and $M(z)$ by expressing $\hat{A}, \hat{B}, \hat{C}, \hat{D}$ in terms of $z$ and using them in Eqs. (7.31)-(7.33) [5, 41].

Figure 7.11a and c show the system window at the input plane $z=0$. This region defines the set of all input signals that can pass through the system without any information loss. Input signals whose space-frequency support lies wholly inside this region will not experience any loss. Similarly, Fig. 7.11b and d show the system window at the output plane $z=d$. This region defines the set of all signals that can be observed at the output of the system. The region in Fig. $7.11 \mathrm{~b}$ is just a propagated version of the region in Fig. 7.11a through the entire optical system. This can be obtained by applying the concatenated LCT matrix $\hat{\mathbf{T}}_{L} \ldots \hat{\mathbf{T}}_{2} \hat{\mathbf{T}}_{1}$ to the space-frequency window at the input plane, to take into account the linear distortion due to the entire optical system (by multiplying the coordinates of each corner of the window with the LCT matrix, as described before).

Just as the concatenation property of transformation matrices allows us to represent the cumulative action of all optical elements present with a single entity, the system window is an equivalent aperture that appropriately transforms and combines the effects of all individual apertures in different domains, into a single space-frequency aperture [37].

The space-frequency (phase-space) window of the system in Fig.7.10a is determined only by the $1 \mathrm{st}, 5 \mathrm{th}, 7 \mathrm{th}$, and 8 th apertures. The other apertures do 
Fig. 7.10 (a) An apertured optical system with input plane at $z=0$ and output plane at $z=2 \mathrm{~m}$ [37]. The horizontal axis is in meters. The lens focal lengths $f_{j}$ in meters and the aperture sizes $\Delta_{j}$ in centimeters are given right above them. (b) and (c) Evolution of $a(z)$ and $M(z)$ as functions of $z \cdot \lambda=0.5 \mu \mathrm{m}$ and $s=0.3 \mathrm{~mm}[5,41]$
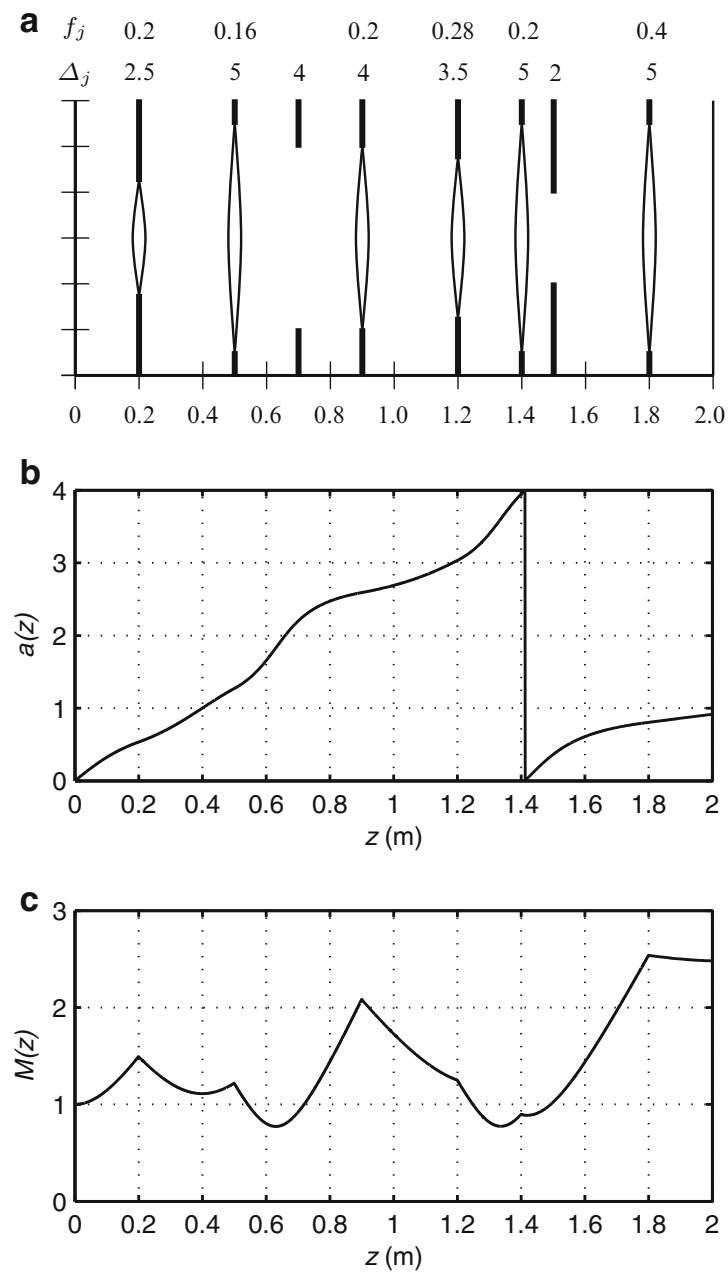

not affect or limit the space-frequency window of the system and therefore can be considered as redundant from the system's viewpoint. (Removing the redundant apertures from the system or replacing them with apertures of greater size will have negligible effect on the behavior of the system, for any given input signal.)

It is also worth noting that the information loss caused by an aperture will depend not only on the actual physical size of the aperture, but also the magnification of the signal at that location. If the magnification at the aperture location is small, there will be less or no information loss. For example, although the aperture sizes are the same for the 2nd, 6th, and 8th apertures, only the 8th aperture limits the system window $(M(1.8) \approx 2.5$ whereas $M(0.5) \approx 1.2$ and $M(1.4) \approx 1)$. This illustrates that the magnification in the plane of the aperture is as important as the size of the aperture in limiting the system window. If we have some flexibility during the 

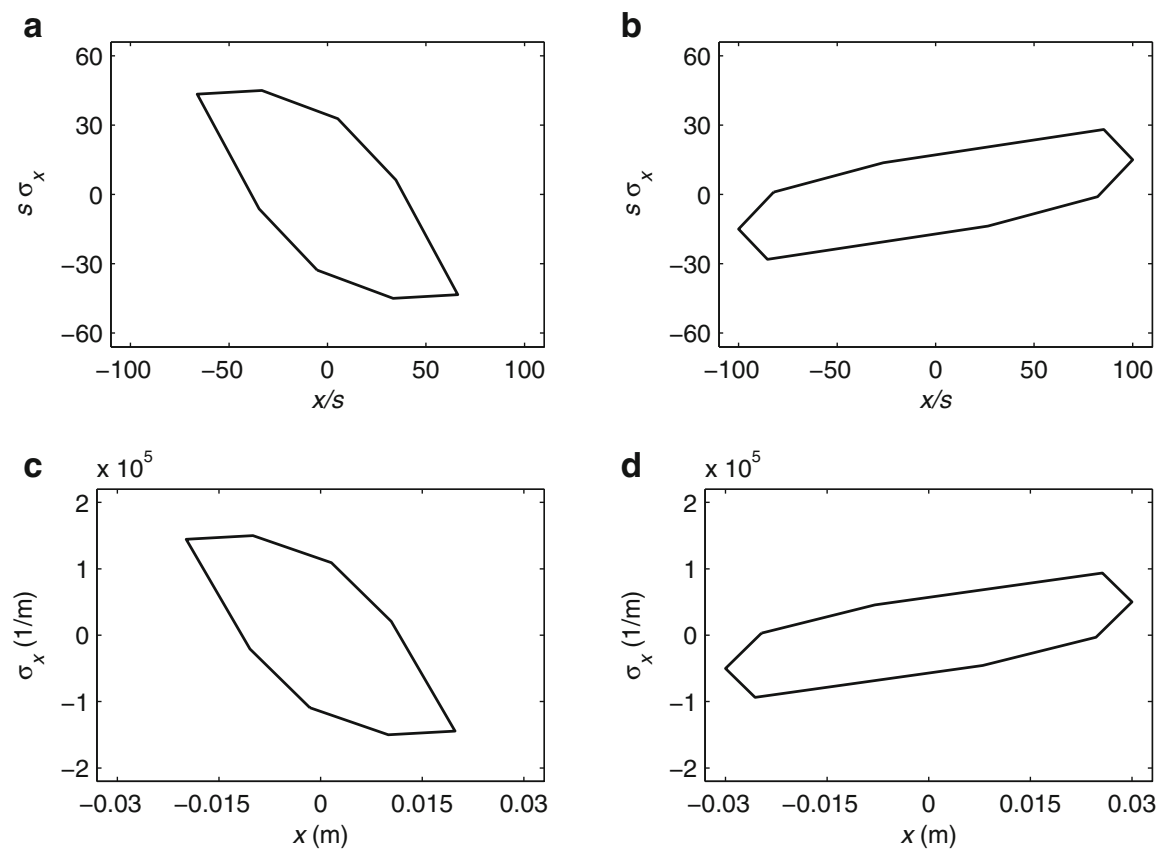

Fig. 7.11 The space-frequency window of the system at the input plane in the dimensionless (a) and dimensional spaces (c). The space-frequency window of the system at the output plane in the dimensionless (b) and dimensional spaces (d) [37]

design of the optical system, careful choice of lens and aperture locations can help information losses to be minimized, a process which will be aided by the spacefrequency approach and the graphs for $M(z)$ we have discussed.

\subsubsection{Necessary and Sufficient Condition for Lossless Transfer}

An input signal will pass through the system without any information loss if and only if its space-frequency support is fully contained in the input space-frequency (phasespace) window of the system. That is, if the signal support does not lie completely inside the system window, information loss will occur [37].

Proof. LCT domains correspond to oblique axes in the space-frequency plane. Consider corridors of varying width, orthogonal to such an oblique axis. The extent of the signal in a given LCT domain can be determined from the space-frequency support of the signal, by finding the width of the narrowest orthogonal corridor enclosing the space-frequency support. First, let us consider an input signal whose space-frequency support lies completely inside the input space-frequency window of the system. This guarantees that along any oblique axis in the space-frequency 
plane corresponding to some LCT domain in which an aperture resides, the extent of the signal will be smaller than the width of the aperture at that LCT domain, and hence the signal will pass through that aperture unhindered. Repeating this for all apertures, the input signal will pass through the whole system without any information loss. (Recall that the space-frequency window is defined by the intersection of the corridors defined by the apertures. If the extent of the signal was larger than the width of the aperture at that LCT domain, the orthogonal corridor enclosing the space-frequency support would have been wider than the corridor defined by the aperture, so that the space-frequency support of the signal could not lie within the space-frequency window of the system.)

Conversely, consider an input signal which passes through the system without any loss. This implies that the signal extent was smaller than the aperture width for each aperture, since otherwise irreversible information loss would occur. Recall that each aperture defines a corridor perpendicular to the LCT domain in which it resides. For any of these LCT domains, the space-frequency support of the signal must lie within this corridor, since if not, the extent of the signal in that domain would not lie within that aperture, leading to information loss and hence a contradiction. Since this argument must be true for all apertures, it follows that the signal space-frequency support must lie inside the region defined by the intersection of the corridors, which is the space-frequency window of the system. This completes the proof.

A straightforward but lengthy way to determine whether information loss will take place would be to trace the space-frequency support of the signal as it passes through the whole system [33]. When the signal arrives at the first aperture, there will have taken place a linear distortion on the initial space-frequency support of the signal. After this linear distortion, if the extent of the signal in that LCT domain is less than the aperture size, then the signal will pass through this aperture without any information loss. Then another linear distortion will take place as the signal travels to the next aperture. Again, we will determine whether there is any information loss by comparing the extent in this domain to the aperture size. Repeating this procedure throughout the system, we can determine whether the signal passes through the system losslessly. This lengthy way of determining whether there will be information loss is specific to a certain input signal and its support. On the other hand, our method is general in the sense that, once the space-frequency window of the system is determined, it specifies the set of all signals that can pass through the system without information loss. The optical system preserves the information content of all signals whose space-frequency support lies inside the space-frequency window of the system.

\subsubsection{Lossy Transfer}

If the space-frequency window does not enclose the space-frequency support of the input signal completely, then we would intuitively expect the following: The 
information contained within the intersection of the space-frequency support of the signal and the space-frequency window of the system will be preserved, and the rest will be lost (Fig. 7.12). This indeed turns out to be approximately true in most cases [37]. In other words, just as a spatial aperture passes certain parts of a signal and blocks the rest, the space-frequency window acts like an aperture in phasespace, passing certain parts and blocking others. In particular, if the set of input signals has a greater number of degrees of freedom than the number of degrees of freedom the system can support, information loss will take place, since a region with larger area can never possibly lie completely within a region with smaller area.

Given an arbitrary space-frequency support at the input, one can obtain the spacefrequency support at any position in the system by tracing the support throughout the system $[33,65]$. Whenever an aperture narrower than the signal extent is encountered, the outlying parts of the signal will be truncated. The effect of this truncation on the space-frequency support of the signal will be to likewise truncate the regions of the support lying outside the corridor defined by the aperture. If this were the only effect of the aperture in the space-frequency plane, then the statements made above would be exact (rather than being approximate) and the space-frequency support observed at the output could simply be found as follows: (a) Find the intersection of the input space-frequency support and the system space-frequency window at the input plane, (b) Propagate this space-frequency region to the output plane. However, this simple and intuitive result is not exact because each aperture that actually cuts off the outlying parts of the signal will also cause a broadening of the support of the signal along the orthogonal domain, due to the Fourier uncertainty relation.

We now argue that the broadening effects are generally negligible for most real physical signals and systems, so that the simple and intuitive result above is usually valid [37]. The effect of an aperture corresponds to multiplication with a rectangle function. Let $\Delta_{j}$ denote the size of the aperture. Firstly, if the signal extent before the aperture is already smaller than $\Delta_{j}$, then the windowing operation will affect
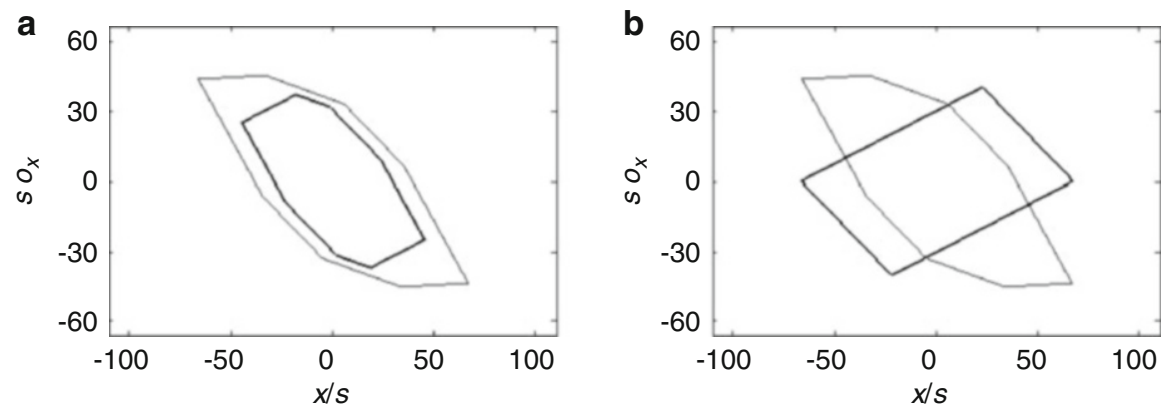

Fig. 7.12 (a) The signal support is wholly contained within the system window so there is no loss of information. (b) The part of the signal support lying within the system window will pass, and the parts lying outside will be blocked [37] 
neither the signal nor its space-frequency support. However, if the signal extent in that domain is larger than $\Delta_{j}$, then the signal will be truncated and the spacefrequency support will also be affected. Because windowing involves multiplication with a rectangle function, it implies convolution of the Wigner distribution of the signal with the Wigner distribution of the rectangle function along the orthogonal direction [44, 59]. (An expression for the Wigner distribution, $\hat{W}_{\text {rect }}\left(x, \sigma_{x}\right)$ of the rectangle function $\operatorname{rect}\left(x / \Delta_{j}\right)$ is known $[5,56]$ but its exact form is not necessary for our argument.) This operation will cause compaction of the Wigner distribution of the signal to a corridor of width $\Delta_{j}$. Moreover, convolving the Wigner distribution of the signal with that of the rectangle function along the orthogonal direction will result in broadening of the Wigner distribution by an amount that is comparable with the extent of $\hat{W}_{\text {rect }}$ in that direction. This extent is approximately $1 / \Delta_{j}$, and thus the spread in the orthogonal direction after windowing will be $\sim 1 / \Delta_{j}[56]$.

For simplicity, consider a rectangular region in the space-frequency plane, in which case the space-bandwidth product can be taken as a measure of the number of degrees of freedom. Let us denote the space-bandwidth product as $N=\Delta x \Delta \sigma_{x} \geq 1$, where $\Delta x$ ve $\Delta \sigma_{x}$ denote the spatial and frequency extents. Noting that the apertures can be modeled as rectangle functions, the frequency extent associated with the rectangle function will approximately be the reciprocal of its spatial extent: $1 / \Delta_{j}$. Let us assume that the aperture extent is a fraction $\kappa$ of the signal extent; that is $\Delta_{j}=\kappa \Delta x$ where $\kappa<1$. After the aperture, the new space-domain signal extent will be given by $\Delta x^{\prime}=\kappa \Delta x$. Moreover, since multiplication in the space-domain implies convolution in the frequency domain, the new extent in the frequency domain will be approximately the sum of the spectral extents of the signal and the aperture. The frequency extent of the signal is $\Delta \sigma_{x}=N / \Delta x$ and the frequency extent of the window is $\sim 1 / \Delta_{j}=1 / \kappa \Delta x=\Delta \sigma_{x} / \kappa N$. Then, the new extent in the frequency domain will be $\Delta \sigma_{x}{ }^{\prime} \approx \Delta \sigma_{x}+\Delta \sigma_{x} / \kappa N=\Delta \sigma_{x}(1+1 / \kappa N)$. Therefore, the space-bandwidth product of the signal after the aperture will be $\Delta x^{\prime} \Delta \sigma_{x}{ }^{\prime} \approx \Delta x \Delta \sigma_{x}(\kappa+1 / N)$. Here, the first term corresponds to the reduced space-frequency support resulting from the truncation inflicted by the aperture, and the second term corresponds to the increase arising from the broadening in the orthogonal direction. However, if $\kappa \gg 1 / N$, or equivalently $N \gg 1 / \kappa$, then we can neglect the term $1 / N$ in comparison with $\kappa$. Thus, we can neglect the broadening effect if $N \gg 1 / \kappa$. This condition will hold for most real physical signals and systems. For a physical signal that contains any reasonable amount of information, such as an image, the number of degrees of freedom will be much larger than unity and also much larger than $1 / \kappa$, as long as $\kappa$ is not very close to 0 . The case where $\kappa$ is very close to 0 is not very likely either, since apertures with very small $\kappa$ truncate nearly all of the signal. For instance, consider a window that allows only 0.1 of the extent of the signal to pass. Even in this case, $N \gg 10$ will be sufficient and most information bearing signals will satisfy this condition easily. Therefore, the broadening effect will be usually negligible when we are dealing with images and other information bearing signals. This in turn means that it is fairly accurate to say that when the space-frequency support of the signal does not wholly lie within the system window, the part that does lie within will pass, and the remaining parts will 
be lost. On the other hand, this simple result will not hold for some signals that do not exhibit too much spatial structure, such as a laser beam, and the broadening effect must be taken into account.

\subsubsection{Discussion and Applications}

We considered optical systems consisting of an arbitrary sequence of lenses and apertures separated by arbitrary lengths of free space (or quadratic graded-index media). We defined the space-frequency window (phase-space window) and showed how it can be explicitly determined for such a system. The area of the window gives the maximum number of degrees of freedom that can be supported by the system. More significantly, the window specifies which signals can pass through the system without information loss; we showed that the signal will pass losslessly if and only if the space-frequency support of the signal lies completely within this window. A precondition for lossless passage is of course that the area of the space-frequency support (and thus the number of degrees of freedom) of the set of input signals must be smaller than the area of the space-frequency window (and thus the number of degrees of freedom the system can support). We further saw that when the spacefrequency support does not lie completely within the space-frequency window, the parts that lie within the window pass and the parts that lie outside of the window are blocked. While the last result is not exact, we showed that it is valid to a good degree of approximation for many systems of practical interest [33, 37].

These results are very intuitive and provide considerable insight and guidance into the behavior and design of systems involving multiple apertures. They can help designing systems in a manner that minimizes information loss, for instance by ensuring that the magnifications are as small as possible at aperture locations. An advantage of this approach is that it does not require assumptions regarding the input signals during analysis or design, since the concept of a system window is signal-independent.

Being able to determine the space-frequency window as a function of the system parameters as we have shown, and the possibility of tailoring and optimizing it has potential applications in areas including optical superresolution [77, 78, 81-85], holographic imaging [75, 80, 86-89], optical encryption systems [90], analysis and design of recording devices [76, 79], and comparison between different implementations of a particular system [91], where apertured optical systems are involved. The system window approach can yield new perspectives and rigorous approaches for such applications and other previously considered problems in the literature.

A potentially important area of application is optical superresolution and spacebandwidth product adaptation [92]. In this area the goal is to adapt the spacefrequency support of the input signal to the space-frequency window of the system based on available a priori information about the signals. In most work in this area, the system window is commonly assumed to be, or approximated as, a rectangular shape or some other simple shape. Being able to precisely calculate the system 
window for a quite broad class of optical systems will make this superresolution approach more accurate, efficient, and widely applicable.

Moreover, in optical encryption [90], the system window can be used to investigate the optimal design of the encryption system, the most efficient representation of encrypted signals, and determination of the number of degrees of freedom that can be encrypted.

Yet another application of the results of this paper is the selection and optimization of recording devices. The system window at the output plane is of special use for this purpose, since it describes the largest space-frequency support that can be observed at the output of the system. This gives the position, maximum spatial extent, maximum frequency bandwidth (or in general the maximum extent in any LCT domain), and maximum space-bandwidth product (or more generally the bicanonical width product) that can be observed at the output of the system. The spatial extent of the system window should be matched to the location and width of the detector to ensure the recording of the entire output. The number of pixels required can be determined by fitting a rectangle to the system window and computing its area. This gives the number of samples needed to reconstruct any output signal from its Nyquist samples. Once the detector width and the number of pixels are determined, the pixel size is also revealed. Such an approach constitutes a new way of analyzing the optical efficiency of detectors [79]. Moreover, if specifications of the detector are pre-determined by some design limitations (such as limited spatial resolution), then the system can be adapted to work as best as it can with the specified detector [60].

The system window can also be useful in comparing alternative implementations of an optical system. One can choose among different implementations by investigating which implementation supports more degrees of freedom and hence causes less information (or power) loss due to the apertures (by comparing the areas of the system windows). Alternatively, the design goal can be to find the system window that is more compatible with the given detector limitations. Such approaches have been pursued, for example, for comparing different holographic systems [89] and different implementations of optical FRTs [91]. However, these previous approaches are either highly dependent on the input signal considered, involve many simplifications to make the analysis feasible, or yield only limited numerical results.

As a final note, we have mostly used the terms space-frequency window or phasespace window to distinguish these entities living in the space-frequency plane, from the physical apertures that act on signals in various LCT (or equivalently FRT) domains. However, since we have seen that these windows block or pass the spacefrequency support of the signal in a manner very similiar to how apertures block or pass the physical signals, we can also speak of space-frequency apertures or phasespace apertures. 


\subsection{Conclusion}

We discussed the relationships between LCT domains, FRT domains, and the spacefrequency plane. In particular, we showed that LCT domains correspond to scaled fractional Fourier domains and thus to scaled oblique axes in the space-frequency plane. This allows LCT domains to be labeled and monotonically ordered by the corresponding fractional order parameter and provides a more transparent view of the evolution of light through an optical system modeled by LCTs.

We then studied the number of degrees of freedom of optical systems and signals based on these concepts. We first discussed the bicanonical width product, which is the number of degrees of freedom of LCT-limited signals. The bicanonical width product generalizes the space-bandwidth product and often provides a tighter measure of the actual number of degrees of freedom of signals. We illustrated the usefulness of the notion of bicanonical width product in two applications: efficient signal representation and efficient system simulation. In the first application we provided a sub-Nyquist sampling approach to represent and reconstruct signals with arbitrary space-frequency support. This approach geometrically amounts to enclosing the support with the smallest possible parallelogram, as opposed to enclosing it with a rectangle as in the classical approach. In the second application we provided a fast DLCT computation method which can accurately compute a (continuous) LCT with the minimum number of samples given by the bicanonical width product. Thus the bicanonical width product is also a key parameter in fast discrete computation of LCTs, and hence in efficient and accurate simulation of optical systems. Given the fundamental importance of the conventional spacebandwidth product in signal processing and information optics, we believe the bicanonical width product will find other applications in these areas as well.

Finally, we focused on the degrees of freedom of optical systems consisting of an arbitrary sequence of lenses and apertures separated by arbitrary lengths of free space (or quadratic graded-index media). We defined the space-frequency window (phase-space window) and showed how it can be explicitly determined for such a system in terms of the system parameters. The area of the window gives the maximum number of degrees of freedom that can be supported by the system. More significantly, the window specifies which signals can pass through the system without information loss; we showed that the signal will pass losslessly if and only if the space-frequency support of the signal lies completely within this window. A precondition for lossless passage is of course that the area of the space-frequency support (and thus the number of degrees of freedom) of the set of input signals must be smaller than the area of the space-frequency window (and thus the number of degrees of freedom the system can support). We further saw that when the spacefrequency support does not lie completely within the space-frequency window, the parts that lie within the window pass and the parts that lie outside of the window are blocked. While the last result is not exact, we showed that it is valid to a good degree of approximation for many systems of practical interest.

Thus, just as the concatenation property of transformation matrices allows us to represent the cumulative action of all optical elements with a single entity, the 
system window is an equivalent aperture that appropriately transforms and combines the effects of all individual apertures in different domains, into a single spacefrequency aperture. These results are very intuitive and provide considerable insight and guidance into the behavior and design of systems involving multiple apertures. For example, they can help designing systems in a manner that minimizes information loss, with the advantage that no assumptions regarding the input signals is required, since the system window is a signal-independent entity. We briefly discussed some potential application areas where the system window approach can yield new perspectives. These include optical superresolution, optical encryption, holographic imaging, design and optimization of recording devices, and comparison of alternative implementations of apertured optical systems.

Acknowledgements This chapter is based on [33-37]. H.M. Ozaktas acknowledges partial support of the Turkish Academy of Sciences.

\section{References}

1. R.K. Luneburg, Mathematical Theory of Optics (University of California Press, Berkeley, 1966)

2. S.A. Collins, Lens-system diffraction integral written in terms of matrix optics. J. Opt. Soc. Am. 60(9), 1168-1177 (1970)

3. M.J. Bastiaans, Wigner distribution function and its application to first-order optics. J. Opt. Soc. Am. 69(12), 1710-1716 (1979)

4. M. Nazarathy, J. Shamir, First-order optics-a canonical operator representation: lossless systems. J. Opt. Soc. Am. 72(3), 356-364 (1982)

5. H.M. Ozaktas, Z. Zalevsky, M.A. Kutay, The Fractional Fourier Transform with Applications in Optics and Signal Processing (Wiley, New York, 2001)

6. K.B. Wolf, Construction and properties of canonical transforms, Chap. 9, in Integral Transforms in Science and Engineering (Plenum Press, New York, 1979)

7. H.M. Ozaktas, O. Aytur, Fractional Fourier domains. Signal Process. 46(1), 119-124 (1995)

8. H. Zhao, Q.-W. Ran, J. Ma, L.-Y. Tan, On bandlimited signals associated with linear canonical transform. IEEE Signal Process. Lett. 16(5), 343-345 (2009)

9. B. Deng, R. Tao, Y. Wang, Convolution theorems for the linear canonical transform and their applications. Sci. China Ser. F Inf. Sci. 49(5), 592-603 (2006)

10. K.K. Sharma, S.D. Joshi, Uncertainty principle for real signals in the linear canonical transform domains. IEEE Trans. Signal Process. 56(7), 2677-2683 (2008)

11. K.K. Sharma, S.D. Joshi, Signal separation using linear canonical and fractional Fourier transforms. Opt. Commun. 265(2), 454-460 (2006)

12. K.K. Sharma, S.D. Joshi, Signal reconstruction from the undersampled signal samples. Opt. Commun. 268(2), 245-252 (2006)

13. K.K. Sharma, New inequalities for signal spreads in linear canonical transform domains. Signal Process. 90(3), 880-884 (2010)

14. B.-Z. Li, R. Tao, Y. Wang, New sampling formulae related to linear canonical transform. Signal Process. 87(5), 983-990 (2007)

15. A. Stern, Uncertainty principles in linear canonical transform domains and some of their implications in optics. J. Opt. Soc. Am. A 25(3), 647-652 (2008)

16. A. Stern, Sampling of compact signals in offset linear canonical transform domains. Signal Image Video Process. 1, 359-367 (2007) 
17. G. Toraldo di Francia, Resolving power and information. J. Opt. Soc. Am. 45(7), 497-499 (1955)

18. D. Gabor, Light and information, in Progress in Optics, vol. I, Chap. 4, ed. by E. Wolf (Elsevier, Amsterdam, 1961), pp. 109-153

19. G. Toraldo di Francia, Degrees of freedom of an image. J. Opt. Soc. Am. 59(7), 799-803 (1969)

20. F. Gori, G. Guattari, Effects of coherence on the degrees of freedom of an image. J. Opt. Soc. Am. 61(1), 36-39 (1971)

21. F. Gori, G. Guattari, Shannon number and degrees of freedom of an image. Opt. Commun. 7(2), 163-165 (1973)

22. F. Gori, G. Guattari, Degrees of freedom of images from point-like-element pupils. J. Opt. Soc. Am. 64(4), 453-458 (1974)

23. F. Gori, S. Paolucci, L. Ronchi, Degrees of freedom of an optical image in coherent illumination, in the presence of aberrations. J. Opt. Soc. Am. 65(5), 495-501 (1975)

24. F. Gori, L. Ronchi, Degrees of freedom for scatterers with circular cross section. J. Opt. Soc. Am. 71(3), 250-258 (1981)

25. L. Ronchi, F. Gori, Degrees of freedom for spherical scatterers. Opt. Lett. 6(10), 478-480 (1981)

26. A. Starikov, Effective number of degrees of freedom of partially coherent sources. J. Opt. Soc. Am. 72(11), 1538-1544 (1982)

27. G. Newsam, R. Barakat, Essential dimension as a well-defined number of degrees of freedom of finite-convolution operators appearing in optics. J. Opt. Soc. Am. A 2(11), 2040-2045 (1985)

28. A.W. Lohmann, Optical Information Processing. Lecture Notes (Optik+Info, Uttenreuth, 1986)

29. F. Gori, Sampling in optics, in Advanced Topics in Shannon Sampling and Interpolation Theory, Chap. 2 (Springer, New York, 1993), pp. 37-83

30. A.W. Lohmann, R.G. Dorsch, D. Mendlovic, Z. Zalevsky, C. Ferreira, Space-bandwidth product of optical signals and systems. J. Opt. Soc. Am. A 13(3), 470-473 (1996)

31. R. Piestun, D.A.B. Miller, Electromagnetic degrees of freedom of an optical system. J. Opt. Soc. Am. A 17(5), 892-902 (2000)

32. R. Solimene, R. Pierri, Number of degrees of freedom of the radiated field over multiple bounded domains. Opt. Lett. 32(21), 3113-3115 (2007)

33. F.S. Oktem, Signal representation and recovery under partial information, redundancy, and generalized finite extent constraints, Master's thesis, Bilkent University, 2009

34. F.S. Oktem, H.M. Ozaktas, Equivalence of linear canonical transform domains to fractional Fourier domains and the bicanonical width product: a generalization of the space-bandwidth product. J. Opt. Soc. Am. A 27(8), 1885-1895 (2010)

35. F.S. Oktem, H.M. Ozaktas, Exact relation between continuous and discrete linear canonical transforms. IEEE Signal Process. Lett. 16(8), 727-730 (2009)

36. F.S. Oktem, H.M. Ozaktas, Degrees of freedom of optical systems and signals with applications to sampling and system simulation, in Imaging and Applied Optics Conference, Optical Society of America, 2013

37. H.M. Ozaktas, F.S. Oktem, Phase-space window and degrees of freedom of optical systems with multiple apertures. J. Opt. Soc. Am. A 30(4), 682-690 (2013)

38. T. Alieva, M.J. Bastiaans, Alternative representation of the linear canonical integral transform. Opt. Lett. 30(24), 3302-3304 (2005)

39. M.J. Bastiaans, T. Alieva, Synthesis of an arbitrary ABCD system with fixed lens positions. Opt. Lett. 31(16), 2414-2416 (2006)

40. J.A. Rodrigo, T. Alieva, M.L. Calvo, Optical system design for orthosymplectic transformations in phase space. J. Opt. Soc. Am. A 23(10), 2494-2500 (2006)

41. H.M. Ozaktas, M.F. Erden, Relationships among ray optical, Gaussian beam, and fractional Fourier transform descriptions of first-order optical systems. Opt. Commun. 143(1-3), 75-86 (1997)

42. H. Ozaktas, S. Arık, T. Coşkun, Fundamental structure of Fresnel diffraction: natural sampling grid and the fractional Fourier transform. Opt. Lett. 36(13), 2524-2526 (2011) 
43. H. Ozaktas, S. Arık, T. Coşkun, Fundamental structure of Fresnel diffraction: longitudinal uniformity with respect to fractional Fourier order. Opt. Lett. 37(1), 103-105 (2012)

44. L. Cohen, Integral Time-Frequency Analysis (Prentice-Hall, Englewood Cliffs, 1995)

45. M.J. Bastiaans, Applications of the Wigner distribution function in optics, in The Wigner Distribution: Theory and Applications in Signal Processing (Elsevier, Amsterdam, 1997), pp. $375-426$

46. G. Forbes, V. Maniko, H. Ozaktas, R. Simon, K. Wolf, Wignerdistributions and phase space in optics. J. Opt. Soc. Am. A 17(12), 2274-2274 (2000)

47. A. Stern, Sampling of linear canonical transformed signals. Signal Process. 86(7), 1421-1425 (2006)

48. J.J. Ding, Research of fractional Fourier transform and linear canonical transform. Ph.D. thesis, National Taiwan University, Taipei, 2001

49. X.-G. Xia, On bandlimited signals with fractional Fourier transform. IEEE Signal Process. Lett. 3(3), 72-74 (1996)

50. A. Zayed, On the relationship between the Fourier and fractional Fourier transforms. IEEE Signal Process. Lett. 3(12), 310-311 (1996)

51. C. Candan, H.M. Ozaktas, Sampling and series expansion theorems for fractional Fourier and other transforms. Signal Process. 83, 1455-1457 (2003)

52. T. Erseghe, P. Kraniauskas, G. Carioraro, Unified fractional Fourier transform and sampling theorem. IEEE Trans. Signal Process. 47(12), 3419-3423 (1999)

53. R. Torres, P. Pellat-Finet, Y. Torres, Sampling theorem for fractional bandlimited signals: A self-contained proof application to digital holography. IEEE Signal Process. Lett. 13(11), 676-679 (2006)

54. R. Tao, B. Deng, W.-Q. Zhang, Y. Wang, Sampling and sampling rate conversion of band limited signals in the fractional Fourier transform domain. IEEE Trans. Signal Process. 56(1), 158-171 (2008)

55. O. Aytur, H.M. Ozaktas, Non-orthogonal domains in phase space of quantum optics and their relation to fractional Fourier transforms. Opt. Commun. 120(3-4), 166-170 (1995)

56. H.M. Ozaktas, B. Barshan, D. Mendlovic, L. Onural, Convolution, filtering, and multiplexing in fractional Fourier domains and their relation to chirp and wavelet transforms. J. Opt. Soc. Am. A 11(2), 547-559 (1994)

57. J.J. Healy, J.T. Sheridan, Cases where the linear canonical transform of a signal has compact support or is band-limited. Opt. Lett. 33(3), 228-230 (2008)

58. H.M. Ozaktas, D. Mendlovic, Fractional Fourier optics. J. Opt. Soc. Am. A 12(4), 743-751 (1995)

59. L. Cohen, Time-frequency distributions-a review. Proc. IEEE 77(7), 941-981 (1989)

60. A. Stern, B. Javidi, Sampling in the light of Wigner distribution. J. Opt. Soc. Am. A 21(3), 360-366 (2004)

61. A. Stern, Why is the linear canonical transform so little known?, in AIP Conference Proceedings (2006), pp. 225-234

62. A. Papoulis, Signal Analysis (McGraw-Hill, New York, 1977)

63. S.-C. Pei, J.-J. Ding, Closed-form discrete fractional and affine Fourier transforms. IEEE Trans. Signal Process. 48(5), 1338-1353 (2000)

64. B.M. Hennelly, J.T. Sheridan, Fast numerical algorithm for the linear canonical transform. J. Opt. Soc. Am. A 22(5), 928-937 (2005)

65. B.M. Hennelly, J.T. Sheridan, Generalizing, optimizing, and inventing numerical algorithms for the fractional Fourier, Fresnel, and linear canonical transforms. J. Opt. Soc. Am. A 22(5), 917-927 (2005)

66. J.J. Healy, B.M. Hennelly, J.T. Sheridan, Additional sampling criterion for the linear canonical transform. Opt. Lett. 33(22), 2599-2601 (2008)

67. J.J. Healy, J.T. Sheridan, Sampling and discretization of the linear canonical transform. Signal Process. 89, 641-648 (2009)

68. H.M. Ozaktas, A. Koç, I. Sari, M.A. Kutay, Efficient computation of quadratic-phase integrals in optics. Opt. Lett. 31(1), 35-37 (2006) 
69. A. Koc, H.M. Ozaktas, C. Candan, M.A. Kutay, Digital computation of linear canonical transforms. IEEE Trans. Signal Process. 56(6), 2383-2394 (2008)

70. J.J. Healy, J.T. Sheridan, Reevaluation of the direct method of calculating Fresnel and other linear canonical transforms. Opt. Lett. 35(7), 947-949 (2010)

71. J.J. Healy, J.T. Sheridan, Fast linear canonical transforms. J. Opt. Soc. Am. A 27(1), 21-30 (2010)

72. H. Ozaktas, O. Arikan, M. Kutay, G. Bozdagi, Digital computation of the fractional Fourier transform. IEEE Trans. Signal Process. 44(9), 2141-2150 (1996)

73. A. Koc, H. Ozaktas, L. Hesselink, Fast and accurate computation of two-dimensional nonseparable quadratic-phase integrals. J. Opt. Soc. Am. A 27(6), 1288-1302 (2010)

74. L. Durak, O. Arikan, Short-time Fourier transform: two fundamental properties and an optimal implementation. IEEE Trans. Signal Process. 51(5), 1231-1242 (2003)

75. A.W. Lohmann, The space-bandwidth product, applied to spatial filtering and holography, Research Paper RJ-438, IBM San Jose Research Laboratory, San Jose, 1967

76. A. Stern, B. Javidi, Shannon number and information capacity of three-dimensional integral imaging. J. Opt. Soc. Am. A 21(9), 1602-1612 (2004)

77. D. Mendlovic, A. Lohmann, Space-bandwidth product adaptation and its application to superresolution: fundamentals. J. Opt. Soc. Am. A 14(3), 558-562 (1997)

78. Z. Zalevsky, D. Mendlovic, A. Lohmann, Understanding superresolution in Wigner space. J. Opt. Soc. Am. A 17(12), 2422-2430 (2000)

79. P. Catrysse, B. Wandell, Optical efficiency of image sensor pixels. J. Opt. Soc. Am. A 19(8), 1610-1620 (2002)

80. J. Maycock, C. McElhinney, B. Hennelly, T. Naughton, J. McDonald, B. Javidi, Reconstruction of partially occluded objects encoded in three-dimensional scenes by using digital holograms. Appl. Opt. 45(13), 2975-2985 (2006)

81. D. Mendlovic, A. Lohmann, Z. Zalevsky, Space-bandwidth product adaptation and its application to superresolution: examples. J. Opt. Soc. Am. A 14(3), 563-567 (1997)

82. K. Wolf, D. Mendlovic, Z. Zalevsky, Generalized Wigner function for the analysis of superresolution systems. Appl. Opt. 37(20), 4374-4379 (1998)

83. Z. Zalevsky, N. Shamir, D. Mendlovic, Geometrical superresolution in infrared sensor: experimental verification. Opt. Eng. 43(6), 1401-1406 (2004)

84. Z. Zalevsky, V. Mico, J. Garcia, Nanophotonics for optical super resolution from an information theoretical perspective: a review. J. Nanophotonics 3(1), 032502-032502 (2009)

85. J. Lindberg, Mathematical concepts of optical superresolution. J. Opt. 14(8), 083001 (2012)

86. L. Xu, X. Peng, Z. Guo, J. Miao, A. Asundi et al., Imaging analysis of digital holography. Opt. Express 13(7), 2444-2452 (2005)

87. M. Testorf, A. Lohmann, Holography in phase space. Appl. Opt. 47(4), A70-A77 (2008)

88. U. Gopinathan, G. Pedrini, B. Javidi, W. Osten, Lensless 3D digital holographic microscopic imaging at vacuum UV wavelength. J. Disp. Technol. 6(10), 479-483 (2010)

89. D. Claus, D. Iliescu, P. Bryanston-Cross, Quantitative space-bandwidth product analysis in digital holography. Appl. Opt. 50(34), H116-H127 (2011)

90. B. Hennelly, J. Sheridan, Optical encryption and the space bandwidth product. Opt. Commun. 247(4), 291-305 (2005)

91. J. Healy, J. Sheridan, Bandwidth, compact support, apertures and the linear canonical transform in ABCD systems, in Proceedings of the SPIE, vol. 6994 (2008), p. 69940W

92. Z. Zalevsky, D. Mendlovic, Optical Superresolution (Springer, New York, 2004) 